\title{
Extent and structure of intervening absorbers from absorption lines redshifted on quasar emission lines ${ }^{\star}$
}

\author{
J. Bergeron and P. Boissé
}

\begin{abstract}
Institut d'Astrophysique de Paris, UPMC - CNRS, UMR 7095, 98 bis Bd Arago, 75014 Paris, France e-mail: bergeron@iap.fr
\end{abstract}

Received 31 March 2017 / Accepted 12 May 2017

\begin{abstract}
Aims. We wish to study the extent and sub-parsec spatial structure of intervening quasar absorbers, mainly those involving cold neutral and molecular gas.

Methods. We have selected quasar absorption systems with high spectral resolution and a good signal-to-noise ratio data, with some of their lines falling on quasar emission features. By investigating the consistency of absorption profiles seen for lines formed either against the quasar continuum source or on the much more extended (Ly $\alpha-\mathrm{N} \mathrm{v}, \mathrm{CIV}$ or Ly $\beta$-O VI) emission line region (ELR), we can probe the extent and structure of the foreground absorber over the extent of the ELR $(\sim 0.3-1 \mathrm{pc})$. The spatial covering analysis provides constraints on the transverse size of the absorber and thus is complementary to variability or photoionisation modelling studies, which yield information on the absorber size along the line of sight. The methods we used to identify spatial covering or structure effects involve line profile fitting and curve-of-growth analysis.

Results. We have detected three absorbers with unambiguous non-uniformity effects in neutral gas. For the extreme case of the Fe I absorber at $z_{\text {abs }}=0.45206$ towards HE 0001-2340, we derive a coverage factor of the ELR of at most 0.10 and possibly very close to zero; this implies an overall absorber size no larger than $0.06 \mathrm{pc}$. For the $z_{\mathrm{abs}}=2.41837 \mathrm{C}$ I absorber towards QSO J1439+1117, absorption is significantly stronger towards the ELR than towards the continuum source in several $\mathrm{CI}$ and $\mathrm{CI}^{\star}$ velocity components, pointing to spatial variations of their column densities of about a factor of two and to structures at the $100 \mathrm{au}-0.1 \mathrm{pc}$ scale. The other systems with firm or possible effects can be described in terms of a partial covering of the ELR, with coverage factors in the range $0.7-1$. The overall results for cold neutral absorbers imply a transverse extent of about five times the ELR size or smaller, which is consistent with other known constraints. Although not our primary goal, we also checked when possible that singly-ionised absorbers are uniform at the parsec scale, in agreement with previous studies. In Tol 0453-423, we have discovered a very unusual case with a small but clearly significant residual flux for a saturated Fe II $\lambda 2600$ line at $z_{\text {abs }}=0.72604$ seen on Ly $\alpha$ emission, thus with an absorber size comparable to or larger than that of the ELR.
\end{abstract}

Key words. ISM: structure - quasars: absorption lines

\section{Introduction}

Some of the absorption systems detected in distant quasar spectra that are commonly used to investigate the properties of diffuse gas in various environments throughout the universe have been noted to display unexpected properties in the relative strength of absorption lines that are associated with distinct transitions from a given species. For instance, in some systems with broad absorption lines (BAL) or in intrinsic systems, the observed resolved profiles of multiplet lines are clearly inconsistent with models in which the absorber uniformly covers the background source (Barlow \& Sargent 1997; Ganguly et al. 1999). This is especially evident when resolved line profiles display a flat core, which indicates saturation together with a non-zero residual flux at the line centre. In BAL systems, such effects can be easily explained by invoking small cloudlets within the disc wind that only partially cover the quasar accretion disc.

A variant of this situation is found when the profile of intervening absorption lines that fall on top of quasar emission features appears to be inconsistent with the profile of lines from the same species that formed against the continuum source alone. One good example is the $\mathrm{C} I$ and $\mathrm{H}_{2}$ system at $z_{\mathrm{abs}}=2.3377$

\footnotetext{
^ Based on observations with VLT-UVES and Keck-HIRES.
}

towards LBQS 1232+082 (Balashev et al. 2011). The C $\mathrm{I} 1656$ feature arises on the C IV quasar emission line; thus, the background flux at the corresponding wavelength is provided by both the accretion disc that is responsible for the continuum emission and by the C IV emission line region (hereafter ELR), while the $\mathrm{C} I \lambda 1560$ line is seen against the continuum source alone. While the accretion disc is no larger than about 100 au (Dai et al. 2010), the ELR is much more extended, with a size in the range $0.1-1 \mathrm{pc}$ for luminous quasars (Bentz et al. 2009). Since the C I $\lambda 1656$ feature is weaker than $C I \lambda 1560$, whereas the opposite is expected on the basis of oscillator strength values, one cannot escape the conclusion that the $\mathrm{CI}$ absorber is not uniform over the whole background source and covers the extended ELR only partially. A few other systems that display effects of this type have been reported (see, e.g., Krogager et al. 2016; Fathivavsari et al. 2017); we note, however, that the interpretation for some of them remains ambiguous because the presence of unresolved optically thick velocity components might be sufficient to explain the observed line ratio.

When present, these effects imply a lower apparent opacity for the lines that are affected by partial covering. This biases the determination of column densities, $N$, and Doppler parameters, $b$. Thus, an immediate objective is to properly take these effects 
into account in order to obtain correct $N$ and $b$ values. A further more essential motivation is related to our knowledge of the size and internal structure of the associated gaseous clouds. In the intervening systems mentioned above, the peculiar relative strength of absorption lines is related to the finite extent of the background source and more specifically to its composite nature, involving two widely different scale lengths $(\approx 100$ au and $1 \mathrm{pc})$. By modelling the profile of absorption lines that fall on or away from quasar emission features, one should then be able to compare the absorber size to these scale lengths.

This approach is especially relevant for molecular or neutral gas (as probed, e.g., by C I lines for high-redshift systems) since cloud sizes in the range $0.1-10 \mathrm{pc}$ have been inferred (see Jenkins \& Tripp 2011 for Galactic gas and Jorgenson et al. 2010 for high-redshift systems), which is comparable to the ELR size. These scales have been derived from the analysis of $\mathrm{C}$ I finestructure transitions, which provides an estimate of the volume density; the inferred extent is therefore along the line of sight. In contrast, the analysis of partial covering effects yields constraints on the transverse size. Thus, both methods provide independent complementary estimates, and their comparison should lead to a robust estimate of the absorber extent, which is a key parameter for modelling.

The absorption lines induced by an absorber located in front of an extended background source is governed not only by their relative extent, but also by the small-scale structure within the intervening gas. In the interstellar medium of our own Galaxy, small-scale structure in the neutral medium (as traced by C I or $\mathrm{NaI}$ ) is observed at all scales above about 10 au (Welty 2007; Watson \& Meyer 1996). If structure over such small scales is also present in high-redshift galaxies, it should manifest itself through time changes in quasar absorption lines, as argued recently by Boissé et al. (2015). Transverse peculiar velocities of a few $100 \mathrm{~km} \mathrm{~s}^{-1}$ are expected for the observer, quasars, and intervening galaxies. This implies drifts of the line of sight through the absorber of tens to hundreds of au over a time interval of about $10 \mathrm{yr}$. To date, only tentative variations ( $3 \sigma$ significance level) have been observed for neutral $(\mathrm{C} \mathrm{I})$ and molecular $\left(\mathrm{H}_{2}\right)$ gas in a damped Ly $\alpha$ (DLA) absorber at $z_{\mathrm{abs}}=2.05454$ towards FBQS J2340-0053 over a two-year time interval (Boissé et al. 2015, and references therein for other approaches involving quasar pairs or lensed quasars). If internal structure were present in the intermediate $100 \mathrm{au}-1 \mathrm{pc}$ range within distant neutral or molecular absorbers, this would potentially affect the behaviour of absorption lines that are detected on quasar emission features. Thus, a detailed analysis of such absorption lines can provide useful information that complements the information provided by time variation studies.

While these effects have been investigated in many quasar intrinsic systems (see, e.g., Hamann et al. 2011), to our knowledge no systematic study has been performed for intervening systems. Only a few cases have been identified, suggesting that these effects are rare, while given the similarity between the size inferred for the C I absorbers and the ELR extent (Jorgenson et al. 2010), one might expect them to be common. In order to clarify this question and derive useful constraints on the extent and smallscale structure of distant neutral absorbers, we have assembled a sample of $\mathrm{C}, \mathrm{Fe} \mathrm{I}$, and $\mathrm{H}_{2}$ systems detected in quasar spectra with a good signal-to-noise ratio $(\mathrm{S} / \mathrm{N})$ and high resolution, and we investigate their properties in a systematic manner.

This paper is organised as follows. In Sect. 2 we describe the various effects that can be expected when an absorber is not uniform over the whole extent of the ELR together with the simplest models that can be used to account for them. We also discuss
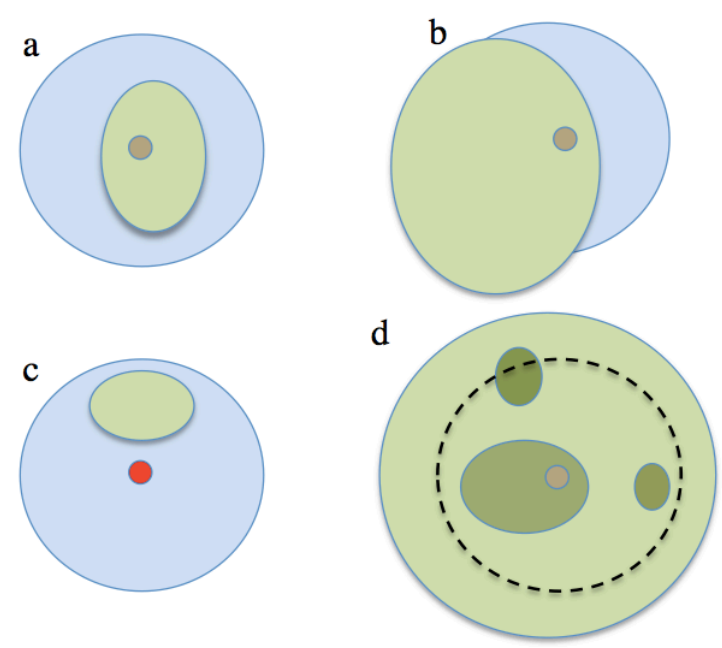

Fig. 1. Four configurations for the quasar light source and foreground absorber. The continuum source (i.e., accretion disc) appears in red (or black when occulted), while the extended emission line region (ELR) is plotted in light blue. The absorber (green) may cover the continuum source, but only part of the ELR $a$ ), b), as assumed in the partial covering model. It may also cover part of the ELR, but not at all the continuum source $c$ ), or, more likely, cover the whole quasar source but in a non-uniform way, if internal structure is present (yellow areas correspond to regions inducing weaker absorption; the black dotted line delineates the ELR). In cases $a$ ) and $b$ ), absorption is stronger towards the continuum source than towards the ELR, whereas it is weaker in cases $c$ ) and $d$ ).

under which conditions the non-uniformity can be established unambiguously. Section 3 presents the sample we studied and the analysis we performed in order to search for partial covering or structure effects. Our results on the transverse extent of the absorbers are presented in Sect. 4. A discussion together with future prospects are given in Sect. 5 .

\section{Effects due to non-uniform absorbers}

\subsection{Expected effects}

When an absorber is not uniform over the extent of the background source, two alternatives are possible. First, the absorption towards the ELR (that is, seen on a quasar emission line) can be weaker than what is expected on the basis of absorption lines that are detected against the continuum source alone. This corresponds to the classical partial covering effect (Figs. 1a,b). In the most extreme situation, the fraction of the ELR that is covered can be so small that the emission line flux remains essentially unabsorbed (we recall that the size of the ELR is at least two orders of magnitude larger than that of the accretion disc).

Second, the absorption seen against the ELR can be stronger than that detected on the continuum; in Figs. 1c and d, we show two configurations corresponding to this alternative. The absorber in Fig. 1c does not absorb the continuum source flux at all and that in Fig. 1d is inhomogeneous, with an average absorption of the emission line flux larger than that of the continuum flux. 


\subsection{Models}

\subsubsection{Partial covering model}

Several authors have proposed models that can be used to describe absorption by a non-uniform gas layer. The most popular is the partial covering model (Barlow \& Sargent 1997; Hamann et al. 1997; Ganguly et al. 1999), in which the absorber is assumed to uniformly cover some fraction of the source with an opacity $\tau(\lambda)$, the remaining fraction being unabsorbed. In this picture, the observed flux writes

$F_{\text {obs }}(\lambda)=C_{\mathrm{f}} F_{\mathrm{b}} \exp (-\tau(\lambda))+\left(1-C_{\mathrm{f}}\right) F_{\mathrm{b}}$,

where $F_{\mathrm{b}}$ is the total background flux $\left(F_{\mathrm{b}}=F_{\mathrm{c}}+F_{\mathrm{elr}}\right.$, with $F_{\mathrm{c}}$ and $F_{\text {elr }}$ referring to the continuum and ELR sources, respectively), $\tau$ is the line opacity and $C_{\mathrm{f}}$ the covering factor, that is, the fraction of the background flux that is covered by the absorber. In this equation, $F_{\mathrm{b}}$ varies smoothly with wavelength over the interval covered by the absorption line. The term $\exp (-\tau(\lambda))$ describes the absorption line profile. The corresponding normalised spectrum is

$F_{\text {obs }, n}(\lambda)=C_{\mathrm{f}} \exp (-\tau(\lambda))+\left(1-C_{\mathrm{f}}\right)$.

An immediate consequence of partial covering is that saturated lines do not reach the zero level, but display a non-zero line flux residual (LFR, after Balashev et al. 2011) at their core. For $\exp (-\tau) \ll 1$, the above relation gives

$F_{\text {obs }, n}($ core $)=\mathrm{LFR}=1-C_{\mathrm{f}}$.

For resolved doublet lines of this type, the apparent opacity ratio is no longer equal to the atomic physics value, but reaches unity when both lines are optically thick and affected by the same $C_{\mathrm{f}}$ value. The LFR can be read directly on the observed spectrum, providing $C_{\mathrm{f}}\left(C_{\mathrm{f}}=1-\mathrm{LFR}\right)$. If lines are not resolved, profile fitting or curve-of-growth analysis of at least two transitions must be used the derive the LFR and $C_{\mathrm{f}}$ values.

$C_{\mathrm{f}}$ is determined by the fraction of the ELR and of the continuum flux that is occulted, $C_{\text {elr }}$ and $C_{\mathrm{c}}$, respectively, and by the ratio of the emission line to the continuum flux, $x=F_{\text {elr }} / F_{\mathrm{c}}$, at the wavelength of the absorption feature considered (we follow the notations introduced by Ganguly et al. 1999, except for $W$, which is commonly used for equivalent widths, for which we adopt $x$ ). From the relation $C_{\mathrm{f}} F_{\mathrm{b}}=C_{\mathrm{elr}} F_{\text {elr }}+C_{\mathrm{c}} F_{\mathrm{c}}$, we obtain

$C_{\mathrm{f}}=\frac{C_{\mathrm{c}}+x C_{\mathrm{elr}}}{1+x}$ or, equivalently, $C_{\mathrm{elr}}=\frac{C_{\mathrm{f}}(1+x)-C_{\mathrm{c}}}{x}$.

The configurations shown in Figs. 1a,b correspond to $C_{\mathrm{c}}=1$ and $C_{\text {elr }}<1$, while $C_{\mathrm{c}}=0$ and $C_{\text {elr }}<1$ correspond to the configuration sketched in Fig. 1c. In practise, the $x$ value can be estimated by interpolating the continuum measured on the blue and red side of the emission feature at the location of the absorption line (this is performed more accurately if a flux-calibrated spectrum is available). We stress that in this model the absorber is uniform (with the advantage of introducing a mininum number of free parameters) but the covering of the source is not.

\subsubsection{Two-value model}

The above picture would not be appropriate to describe absorbers in which the opacity towards the continuum source is lower than the value averaged over the entire background source, as in Fig. 1d. In this case, one could instead consider a two-value model involving distinct opacities for the gas in front of the continuum source, $\tau_{\mathrm{c}}(\lambda)$, and in front of the ELR, $\tau_{\mathrm{elr}}(\lambda)$ (note that
Ganguly et al. 1999 considered a sort of mixed model in their Appendix A2, involving two opacity values $\tau_{\text {elr }}$ and $\tau_{\mathrm{c}}$ together with two covering factors $C_{\mathrm{c}}$ and $C_{\mathrm{elr}}$ ). With the notations introduced above, the observed profile writes

$F_{\text {obs }}(\lambda)=F_{\mathrm{c}} \exp \left(-\tau_{\mathrm{c}}(\lambda)\right)+F_{\mathrm{elr}} \exp \left(-\tau_{\mathrm{elr}}(\lambda)\right)$,

corresponding to the normalised profile,

$F_{\mathrm{obs}, n}(\lambda)=\frac{\exp \left(-\tau_{\mathrm{c}}(\lambda)\right)+x \exp \left(-\tau_{\mathrm{elr}}(\lambda)\right)}{1+x}$.

This model, with only two discrete opacity values, $\tau_{\mathrm{c}}$ in front of the continuum source and $\tau_{\text {elr }}$ elsewhere may look academic (we note that the partial covering model also assumes two discrete values, $\tau$ and 0 , but their spatial distributions are different in the two models). A more realistic picture is that of an absorber displaying spatial fluctuations of the opacity that are due to internal structure within the region probed by the ELR. If the fluctuations remain moderate enough, one may adequately represent the inhomogeneous gas layer by the two parameters $\tau_{\mathrm{c}}$ and $\tau_{\text {elr }}$, where $\tau_{\text {elr }}$ characterises the "effective" (i.e., "equivalent uniform") absorber intercepted by the ELR. If in this picture the continuum source is located behind a region of low (respectively large) column density, this will result in $\tau_{\text {elr }}>\tau_{\mathrm{c}}$ (respectively $\tau_{\text {elr }}<\tau_{\text {c }}$ ), and the $\tau_{\text {elr }} / \tau_{\text {c }}$ ratio can be used as a measure of the deviation from a uniform covering. This two-value model is more flexible than the partial covering picture and can potentially describe a wider range of physical situations. Since the underlying assumptions about the geometry are distinct in these types of models, there is no rigorous correspondence between them. In the optically thin limit, however, one can obtain the $\tau_{\mathrm{c}}$ and $\tau_{\text {elr }}$ values corresponding to a given partial covering model (characterised by $\tau$ and $C_{\mathrm{f}}$ ) by using Eqs. (2) and (5) and by setting $C_{\mathrm{c}}=1$ as well as $\tau_{\mathrm{c}}=\tau$ (Eqs. (2) and (5), which do not have the same functional form in general, become equivalent in the $\tau \ll 1$ limit). As expected, we obtain $\tau_{\text {elr }}=C_{\text {elr }} \tau$, which is the average opacity value over the ELR extent.

If appropriate absorption lines formed against the continuum source can be used to derive $\exp \left(-\tau_{\mathrm{c}}(\lambda)\right)$, then in the frame of this model, Eq. (6) provides the absorption profile that one would obtain against the ELR alone,

$\exp \left(-\tau_{\mathrm{elr}}(\lambda)\right)=\frac{(1+x) F_{\mathrm{obs}, n}-\exp \left(-\tau_{\mathrm{c}}(\lambda)\right)}{x}$,

which potentially allows us to derive $\tau_{\text {elr }}$ and next to compare it to $\tau_{\mathrm{c}}$. We note that the above relations are still valid if the convolution by the instrumental line spread function is taken into account.

We finally mention that some other non-uniform models such as the power-law model have been introduced by Arav et al. (2005) in the context of intrinsic absorbers.

\subsection{Analysis of absorption lines on quasar emission features}

In practise, most of the absorption lines from the systems considered in this paper are only partially spectroscopically resolved or unresolved. Furthermore, they often involve blends of adjacent velocity components for a given transition or blends of various transitions (e.g., those from $\mathrm{CI}$ and $\mathrm{CI}^{\star}$ ). Thus, we need to rely on a line fitting procedure; we used VPFIT10.2 ${ }^{1}$ for this purpose, and we adopted the oscillator strength $f$ values of Morton (2003). If for a given system there is more than one

1 http://wWw . ast.cam.ac.uk/ rfc/vpfit.html 
transition seen against the quasar continuum, they were analysed together to obtain a fit of the normalised spectrum under the assumption of a uniform covering of the background source. This fit $\left(F_{\text {fit }}(\lambda)\right)$ was then compared to the profile that is observed for the transitions that fall on emission lines. If the latter appear weaker than the prediction drawn from the fit, the partial covering model (with $C_{\mathrm{f}}<1$ ) can be used for these transitions. The associated corrected synthetic profile writes

$F_{\text {fit,corr }}(\lambda)=C_{\mathrm{f}} F_{\text {fit }}(\lambda)+\left(1-C_{\mathrm{f}}\right)$.

The optimum $C_{\mathrm{f}}$ value, or a lower bound when no evidence of $C_{\mathrm{f}}<1$ is found, can be obtained by minimizing the $\chi^{2}$ computed from the difference between the observed and corrected synthetic profiles.

Often, too few lines arising on the continuum alone are available; we therefore simultaneously fit the profile of all transitions that are observed, against both the continuum source and the ELR. We first assume $C_{\mathrm{f}}=1$ for all lines; if the fit is not satisfactory with, for instance, absorption lines seen on an emission feature that are overfitted, we consider $C_{\mathrm{f}}<1$ values for these transitions. In this case, the simultaneous fit must be performed after rescaling the observed spectrum near the corresponding lines to account for the fact that only a fraction $C_{\mathrm{f}}$ of the flux is affected by absorption:

$F_{\text {corr }, n}(\lambda)=\frac{F_{\mathrm{obs}, n}(\lambda)-\left(1-C_{\mathrm{f}}\right)}{C_{\mathrm{f}}}$.

Again, the optimum $C_{\mathrm{f}}$ value can be obtained by $\chi^{2}$ minimization (in this case, all transitions are used to compute $\chi^{2}$ ).

If the opposite applies and absorption lines detected on quasar emission features appear to be underfitted, the two-value model must be adopted. Once the absorption profiles towards the ELR have been derived from Eq. (6), they can be directly fitted, provided at least two distinct transitions occurring on emission lines are available. This leads to a separate determination of $N$ and $b$ parameters for the gas toward the continuum source and ELR (this case is illustrated in Sect. 3.2.8 by the C I system at $z_{\mathrm{abs}}=2.41837$ in QSO J1439+1117).

The curve-of-growth approach can also be used if the absorption involves well-detached velocity components and if no blending of distinct transitions is present. The usual curveof-growth method implicitly assumes uniform covering of the source. In the presence of partial covering, it follows directly from Eq. (2) that the equivalent width $W^{\prime}$ becomes

$W^{\prime}=\int\left(1-F_{\text {obs, } \mathrm{n}}(\lambda)\right) \mathrm{d} \lambda=C_{\mathrm{f}} \int\left(1-e^{-\tau(\lambda)}\right) \mathrm{d} \lambda=C_{\mathrm{f}} W$

where $W$ is the value that one would obtain if the source were fully covered. Alternatively, in the framework of the two-value model, Eq. (6) leads to

$W^{\prime}=\frac{W_{\mathrm{c}}+x W_{\mathrm{elr}}}{1+x}$,

where $W_{\mathrm{c}}$ and $W_{\text {elr }}$ are the equivalent widths that one would measure separately towards the continuum and ELR sources, respectively ( $W^{\prime}$ is just the flux-weighted average, as expected). If enough transitions are available to define a curve of growth for the absorber situated in front of the continuum source (i.e., $W_{\mathrm{c}}$ values), the location of observed $W^{\prime}$ values for transitions that fall on quasar emission lines relative to this curve will indicate whether the absorption formed against the ELR is weaker or stronger than that against the continuum source $\left(W^{\prime}<W_{\mathrm{c}}\right.$, thus $W_{\text {elr }}<W_{\mathrm{c}}$ in the former case and $W^{\prime}>W_{\mathrm{c}}$, thus $W_{\text {elr }}>W_{\mathrm{c}}$ in the latter).

\subsection{Reliable identification of non-uniformity effects}

We now discuss in which conditions it is possible to unambiguously establish the reality of non-uniformity effects in a quasar absorption system. An obvious difficulty comes from the fact that partial covering of the background source and components with unresolved saturated features can both affect line profiles or equivalent width ratios in the same way.

We first consider two lines from the same species detected on a quasar emission feature and examine whether their relative strength can be used to assess non-uniform covering. If the lines are close to each other in the spectrum (as in the case of a $\mathrm{Mg}$ II doublet, for instance) and remain unresolved, both equivalent widths will be affected in a similar way (the two lines are characterised by the same $x$ and hence $C_{\mathrm{f}}$ values) and their ratio is unchanged. The situation is more favorable when lines are fully resolved, especially if some display a flat-bottom profile, leading to a direct determination of the LFR and then of $C_{\mathrm{f}}$ (cf. Eq. (3)). Using at least two lines is important to check that the flat bottom is not due to a blend of several unresolved velocity components, even though this is unlikely when the flat core is extended enough because an unrealistic combination of widths, opacities, and component separations would be required to produce a flat profile. There are cases of intervening systems for which the LFR is close to, but different from, zero: this was first reported for $\mathrm{H}_{2}$ lines (Balashev et al. 2011; see also Sect. 3.3), and we have discovered this effect for Fe II lines (see Sect. 3.2.4).

Systems in which one line (line 1) is seen against the continuum source and the other (line 2) over an emission feature provide much better constraints, especially for systems involving (generally unresolved) lines from neutral or molecular gas, which are the main motivation for this paper. In the partial covering model, the equivalent widths are $W_{1}^{\prime}=W_{1}$ (we assume $\left.C_{\mathrm{c}}=1\right)$ and $W_{2}^{\prime}=C_{\mathrm{f}} W_{2}$, where $W_{1}$ and $W_{2}$ are the equivalent width values expected for a fully covered source, and their ratio is

$r^{\prime}=\frac{W_{2}^{\prime}}{W_{1}^{\prime}}=C_{\mathrm{f}} \frac{W_{2}}{W_{1}}$.

If instead line 1 were falling on the emission line, these relations would write $W_{1}^{\prime}=C_{\mathrm{f}} W_{1}, W_{2}^{\prime}=W_{2}$, and $r^{\prime}=\frac{1}{C_{\mathrm{f}}} \frac{W_{2}}{W_{1}}$. To illustrate the behaviour of $r^{\prime}$, we consider the $\mathrm{C} \mathrm{I} \lambda 1560$ and $\mathrm{C} \mathrm{I} \lambda 1656$ transitions; depending on $z_{\mathrm{abs}}$ and $z_{\mathrm{em}}$, these lines can be seen with either $\mathrm{C} I \lambda 1560$ or $\mathrm{C} I \lambda 1656$ appearing on the quasar C IV emission line (see Sect. 3.2.10 for such a case). Assuming an emission line to continuum flux ratio, $x=0.5$, we plot in Fig. 2 the variation of $r^{\prime}$ with $C_{\mathrm{f}}$ for Gaussian velocity components with various opacity values, $\tau(\mathrm{C} \mathrm{I} \lambda 1560)$. The useful part of the diagram corresponds to $C_{\mathrm{f}}>\frac{1}{1+x}=0.667$, the minimum value reached when $C_{\text {elr }} \rightarrow 0$. For full covering, the $r^{\prime}=W^{\prime}(\mathrm{C}$ I $\lambda 1656) / W^{\prime}(\mathrm{C}$ I $\lambda 1560)$ ratio must lie in the range $r_{\min }^{\prime}<r^{\prime}<r_{\max }^{\prime}$ with $r_{\min }^{\prime}=1.061$ (the optically thick limit, for which $W$ scales as $\lambda$ ) and $r_{\max }^{\prime}=2.169$ (the thin limit: $W \propto \lambda^{2} f$ ), which remains true even if unresolved components are present. Thus, if $r^{\prime}$ values that fall outside this interval are measured, this is necessarily a signature of non-uniform covering. As can be seen in Fig. 2, $r^{\prime}<r_{\text {min }}^{\prime}$ can be observed for relatively opaque lines when $\mathrm{C} \mathrm{I} \lambda 1656$ appears on the emission line (thick blue lines), whereas $r^{\prime}>r_{\max }^{\prime}$ is found for moderate opacities when $\mathrm{C} I \lambda 1560$ coincides with the emission. A similar plot could be made in the two-value model, using $\tau_{\text {elr }} / \tau_{\mathrm{c}}$ to quantify the non-uniformity of the absorber in front of the quasar source. For each transition $i(i=1$ or 2$)$, one can define the equivalent widths $W_{\mathrm{c}, \mathrm{i}}$, and $W_{\mathrm{elr}, \mathrm{i}}$; the $r^{\prime}$ ratio takes the 


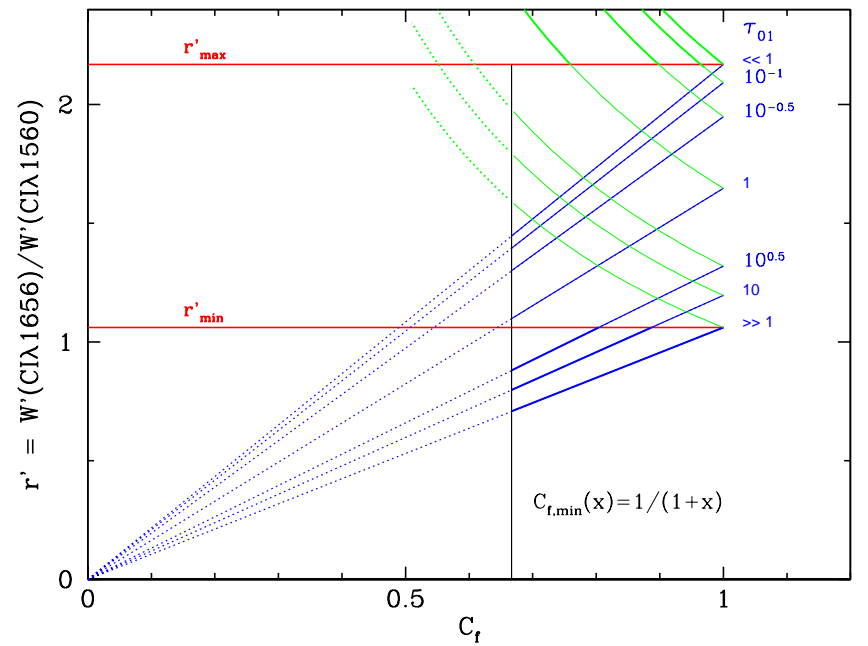

Fig. 2. Variation in equivalent width ratio $r^{\prime}=W^{\prime}(\mathrm{C} \mathrm{I} \lambda 1656) /$ $W^{\prime}(\mathrm{C} \mathrm{I} \lambda 1560)$ with $C_{\mathrm{f}}$ for several values of the $\mathrm{C} \mathrm{I} \lambda 1560$ opacity (indicated in the upper right of the plot) and assuming $x=0.5$. Blue and green lines correspond to $\mathrm{C} I \lambda 1656$ or $\mathrm{C} \mathrm{I} \lambda 1560$, respectively, coinciding with the quasar emission line. The part of the plot for which $C_{\mathrm{f}}<C_{\mathrm{f}, \min }$, assuming $C_{\mathrm{c}}=1.0$, is unphysical (dotted lines). Thick portions of lines correspond to values of $r^{\prime}$ that can be exclusively obtained as a result of non-uniform covering.

form

$r^{\prime}=\frac{W_{2}^{\prime}}{W_{1}^{\prime}}=\frac{W_{\mathrm{c}, 2}}{W_{\mathrm{c}, 1}} \frac{1+x \frac{W_{\mathrm{cll}, 2}}{W_{\mathrm{c}, 2}}}{1+x}$,

assuming that line 2 falls on the quasar emission line. $\frac{W_{\mathrm{elr}, 2}}{W_{\mathrm{c}, 2}}$ can be inferred from the $\tau_{\text {elr }} / \tau_{\mathrm{c}}$ value, provided some assumption is made about the respective $b$ values for the gas lying in front of the ELR and continuum source (e.g., $b_{\mathrm{elr}}=b_{\mathrm{c}}$ ).

Still better constraints can be obtained when several transitions are available to model the absorption profile towards the continuum source. An ideal - but rare - situation occurs when two distinct transitions from the same species are seen on quasar emission lines, allowing us to check the consistency of the analysis, especially if $C_{\mathrm{f}}<1$ or $\tau_{\mathrm{elr}} \neq \tau_{\mathrm{c}}$.

\section{Absorber sample and analysis}

\subsection{Sample of quasars and their absorption systems}

The UVES and HIRES data for the quasars we selected to investigate spatial covering of intervening absorbers are all public and have high S/N spectra: UVES (Bergeron et al. 2004; Molaro et al. 2013) and HIRES (Prochaska et al. 2007).

First, we selected quasar absorption systems that trace molecular and cold neutral gas; the species we considered are mainly $\mathrm{H}_{2}, \mathrm{CI}$, and Fe I. The systems of greatest interest are those where some absorption lines fall on the background quasar emission lines and for species with enough transitions to provide good constraints for line profile fitting.

We then extended our study to a few systems involving moderately ionised gas, mainly C II, Fe II, Ni II, and Si II, for which we wish to check for the absence of substructure at pc scales. Finally, we included the few cases for which the interstellar medium, local or at low redshift, could be studied by its Ca II absorption falling on Ly $\alpha$ or C IV quasar emission lines.

The quasars under investigation are listed in Table 1. Hereafter, the concordance cosmological model is adopted.
Table 1. The sample.

\begin{tabular}{lccrc}
\hline \hline $\begin{array}{l}\text { Target } \\
\text { name }^{a}\end{array}$ & $z_{\mathrm{em}}$ & Spectrograph & $\begin{array}{r}\Delta t \\
\mathrm{~h}\end{array}$ & Date \\
\hline HE 0001-2340 & 2.280 & UVES & 12.0 & $06-08 / 2001$ \\
& & UVES & 15.0 & $09 / 2009$ \\
\hline PKS 0237-23 & 2.225 & UVES & 25.3 & $2001-2002$ \\
& & UVES & 18.8 & $2011-2013$ \\
\hline Tol 0453-423 & 2.261 & UVES & 16.2 & $01 / 2002$ \\
& & UVES & 16.8 & $03-11 / 2011$ \\
\hline TXS 1331+170 & 2.089 & UVES & 8.5 & $03-04 / 2011$ \\
& & HIRES(>4220 A) & 10.0 & $04-06 / 1994$ \\
\hline QSO J1439+1117 & 2.583 & UVES & 8.2 & $03 / 2007$ \\
\hline PKS 1448-232 & 2.208 & UVES & 13.5 & $06-07 / 2001$ \\
\hline FBQS J2340-0053 & 2.085 & UVES & 7.5 & $10 / 2008$ \\
& & HIRES & 4.2 & $08 / 2006$ \\
\hline
\end{tabular}

Notes. ${ }^{(a)}$ Resolved by SIMBAD.

\subsection{Metal systems with absorption line(s) on the quasar Ly $\alpha$ or CIV emission lines}

In this subsection, we successively describe metal systems involving either neutral gas (as traced by $\mathrm{CI}$, Fe I, and Ca II) or moderately ionised gas. Systems involving diffuse molecular gas are considered in Sect. 3.3.

All the systems are listed in Table 2 with the constraints derived on the coverage factors $C_{\mathrm{f}}$ and $C_{\mathrm{elr}}$.

\subsubsection{HE 0001-2340: the Call system at $z_{\text {abs }}=0.2705$}

The CaII 23934,3969 doublet falls on the blue wing of the $\mathrm{C}$ IV emission line. A differential covering effect is thus possible since the ratios of the emission line to the quasar continuum flux differ for the two transitions: $x(\mathrm{Ca}$ II $\lambda 3934)=0.36$ and $x(\mathrm{Ca} I \mathrm{II} 3969)=0.86$. This is a simple system, with a main component of moderate strength at $z_{\mathrm{abs}}=0.270515$, partly resolved, and a very weak component blueshifted by $-19.4 \mathrm{~km} \mathrm{~s}^{-1}$. The fit is good and there is no suggestion of any spatial covering effect; for the main component we obtain $N(\mathrm{Ca}$ II $)=$ $(3.71 \pm 0.12) \times 10^{11} \mathrm{~cm}^{-2}$ and $b=3.38 \pm 0.35 \mathrm{~km} \mathrm{~s}^{-1}$.

In the red part of the spectrum, the associated $\mathrm{NaI} \lambda 5891$, 5897 doublet is not detected. In the Ly $\alpha$ forest, there is an associated strong multiple component $\mathrm{Mg}$ II absorption doublet as well as a Fe II 22586,2600 doublet (partly blended).

\subsubsection{HE 0001-2340: the Fe I system at $z_{\text {abs }}=0.45206$}

This very peculiar absorber has been studied by D'Odorico (2007) and Jones et al. (2010), with extensive photoionisation modelling. There are associated absorptions by rare neutral species, Si I and Ca I, and these authors concluded that this neutral gas system traces a cold medium $(T \lesssim 100 \mathrm{~K})$ of high density $\left(n_{H} \sim 30-1000 \mathrm{~cm}^{-3}\right)$. There are two available UVES spectra, taken about eight years apart (see Table 1); they both have very $\operatorname{good} \mathrm{S} / \mathrm{N}$, with a higher $\mathrm{S} / \mathrm{N}$ redwards of Ly $\alpha$ emission for the 2001 spectrum. Our analysis is based on the latter, which was also the spectrum used by D'Odorico and Jones et al. in their studies of this system. 
Table 2. Systems with absorption line(s) on the quasar Ly $\alpha$ or C IV emission.

\begin{tabular}{lcccccccc}
\hline \hline $\begin{array}{l}\text { Target } \\
\text { name }\end{array}$ & $z_{\mathrm{em}}$ & $z_{\text {abs }}$ & Element & $\begin{array}{c}\text { Number } \\
\text { transitions }\end{array}$ & $\begin{array}{c}\text { Number } \\
\text { components }\end{array}$ & $\begin{array}{c}\text { Structure } \\
\text { effects }\end{array}$ & $C_{\mathrm{f}}$ & $C_{\text {elr }}$ \\
\hline HE 0001-2340 & 2.280 & 0.27052 & Ca II & 2 & 2 & no & 1.0 & 1.0 \\
& & 0.45206 & Fe I & 5 & $1+1^{a, b}$ & yes & $0.30-0.37$ & $0.0-0.10$ \\
& 0.45206 & Mg II & 2 & 1 & possible & $0.75-1.0$ & $0.48-1.0$ \\
\hline PKS 0237-23 & 2.225 & 1.36469 & C I & 2 & 5 & possible & $0.80-0.90$ & $0.71-0.85$ \\
& & 1.36469 & Fe II & 5 & 13 & no & 1.0 & 1.0 \\
\hline Tol 0453-423 & 2.261 & 0.72604 & Fe II & 2 & $5+4$ & yes & 0.98 & 0.96 \\
& & 0.72604 & Mn II & 3 & 5 & no & 1.0 & 1.0 \\
\hline TXS 1331+170 & 2.089 & 0.74461 & Fe I & 5 & 1 & possible & $0.80-1.0$ & $0.56-1.0$ \\
& & 1.32828 & Fe II & 6 & 9 & no & 1.0 & 1.0 \\
& & 1.77653 & C I & 3 & $2+1^{b}$ & possible & $0.90-1.0$ & $0.66-1.0$ \\
\hline QSO J1439+1117 & 2.583 & 2.41837 & C I & 5 & 7 & yes & see & text \\
\hline PKS 1448-232 & 2.208 & -0.00002 & Ca II & 2 & 10 & no & 1.0 & 1.0 \\
\hline FBQS J2340-0053 & 2.085 & 2.05454 & C I & 5 & 8 & yes & $0.85-0.90$ & $0.63-0.75$ \\
\hline
\end{tabular}

Notes. ${ }^{(a)}$ Blended components. ${ }^{(b)}$ Additional component either very weak or noisy.

To estimate the column density $N$ and line width $b$ of the $z_{\mathrm{abs}}=0.45206 \mathrm{Fe}$ I absorber, we have selected the three stronger well-detected transitions that fall on the quasar continuum in the UVES 2001 spectrum: Fe I $\lambda 3021,3720$ redwards of Ly $\alpha$ emission, and $\mathrm{Fe} I \lambda 2484$ (unblended line) in the Ly $\alpha$ forest. A good fit is achieved with a single, unresolved component together with full coverage $C_{\mathrm{f}}=C_{\mathrm{c}}=1$, and we get $N(\mathrm{Fe} \mathrm{I})=$ $(1.95 \pm 0.25) \times 10^{12} \mathrm{~cm}^{-2}$ and $b=0.55 \pm 0.05 \mathrm{~km} \mathrm{~s}^{-1}$.

There is a very weak Fe I $\lambda 2719$ absorption that falls on the blue side of Ly $\alpha$ emission, in a region where a blend of three Ly $\alpha$ absorptions $\left(z_{\mathrm{abs}}=2.24825,2.24876\right.$ and 2.24924$)$ is present together with, bluewards, one Si II $\lambda 1526\left(z_{\text {abs }}=1.58643\right)$ absorption. A plot of the normalised spectrum of this region is shown in Fig. 3, with a fit of the three Ly $\alpha$ and the Si II absorptions. The Fe I $\lambda 2719$ absorption is unexpectedly very weak, although its oscillator strength $f$ is similar to that of Fe $\mathrm{I} \lambda 3021$ (see Fig. 4). This points towards a strong spatial covering effect for this Fe I absorber.

To estimate the spatial coverage factor $C_{\mathrm{f}}$, we renormalised the spectrum around the $\mathrm{Fe} \mathrm{I} \lambda 2719$ line, taking the Ly $\alpha$ and Si II absorptions mentioned above into account to derive the local continuum in this region. This procedure is legitimate because Ly $\alpha$ forest clouds are known to be very large and to display no internal structure at scales comparable to the ELR extent (Rauch et al. 2001). We then used the values of $N$ and $b$ as determined with the three transitions falling on the quasar continuum to fit the renormalised spectrum around the Fe I $\lambda 2719$ line for different values of $C_{\mathrm{f}}$; the best values of $C_{\mathrm{f}}$ correspond to the minimum of $\chi^{2}$. It should be noted that the $\chi^{2}$ of the fit is sensitive to values of the continuum rms, the latter being inversely proportional to $C_{\mathrm{f}}$ (cf. Eq. (9)). To minimize the effect of the continuum noise on the $\chi^{2}$ of the fit around the Fe I $\lambda 2719$ absorption, we therefore limited the selected wavelength range around this line since the $C_{\mathrm{f}}$ values are low. We then obtained $C_{\mathrm{f}}=0.32$ and estimate that the uncertainty on this value is about 0.05 .

The determination of the coverage factor of the emission line region $C_{\text {elr }}$ requires an estimate of the quasar continuum flux $F_{\mathrm{c}}$ underlying the Ly $\alpha$ emission line. There is no low-resolution spectrum of this quasar available at any epoch. Since variability of $\operatorname{Ly} \alpha$ emission flux is detected even in high-redshift quasars

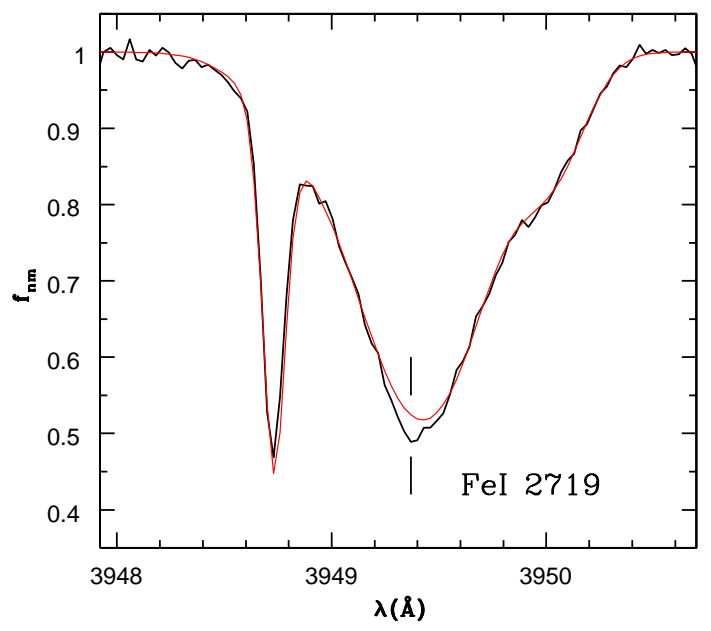

Fig. 3. Intervening Fe $\mathrm{I} \lambda 2719$ absorption at $z_{\mathrm{abs}}=0.45206$ (as marked) towards the quasar HE 0001-2340. This line falls on the blue wing of the quasar Ly $\alpha$ emission line. The spectrum (black curve) is shown together with a fit (see Sect. 3.2.3) of three blended Ly $\alpha$ absorptions, and bluewards, one Si II absorption at $z_{\text {abs }}=1.58643$ (red curve). The $\mathrm{Fe} \mathrm{I} \lambda 2719$ absorption is unexpectedly very weak (see Fig. 4).

(see, e.g., Woo et al. 2013: SDSS data), we used the 2001 UVES spectrum itself, which was taken in good seeing and clear sky conditions, to measure $F_{\mathrm{c}}$ and accordingly the flux ratio $x$. We obtained $x=2.31$, which implies $C_{\mathrm{f}, \min }=0.30$ when assuming full coverage of the quasar continuum $\left(C_{\mathrm{c}}=1 \mathrm{in} \mathrm{Eq.} \mathrm{(4)).} \mathrm{Thus}\right.$ the coverage factor of the ELR is very small: it is consistent with zero, while the coverage factor of the quasar continuum is fully compatible with $C_{\mathrm{c}}=1$. The maximun possible value of $C_{\mathrm{elr}}$ is determined by $C_{\mathrm{f}, \max }=0.37$ and equals $C_{\mathrm{elr}, \max }=0.10$.

The Fe I curve of growth is shown in Fig. 5, adopting the $b$ and $\log N$ values derived from the three transitions seen on the continuum alone. Some weak lines have been included in addition to those used to obtain the fit displayed in Fig. 4 (Fe I $\lambda 2984$ and $\mathrm{Fe} \mathrm{I} \lambda 3841$ together with $\mathrm{Fe} \mathrm{I} \lambda 3441)$ in order to better sample the low-opacity end of the curve. The Fe I $\lambda 2719$ line clearly falls below the curve of growth: the inferred $C_{\mathrm{f}}$ value, $C_{\mathrm{f}}=0.33$, 


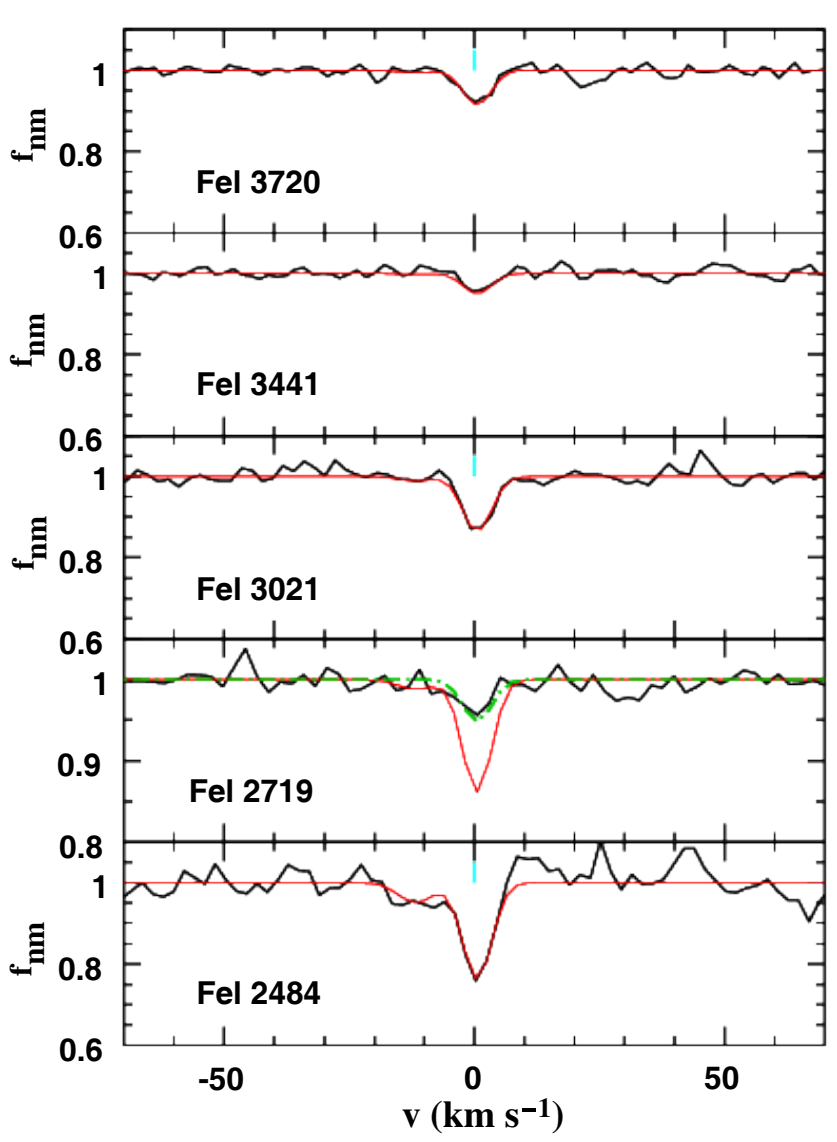

Fig. 4. Intervening Fe I absorption towards the quasar HE 0001-2340: UVES 2001 spectrum (black curve) and simultaneous fit for the four transitions falling on the quasar continuum (red curve), thus excluding the $\mathrm{Fe}$ I $\lambda 2719$ line, which falls on the quasar $\operatorname{Ly} \alpha$ emission line. The green curve for the $\mathrm{Fe} I \lambda 2719$ absorption corresponds to a fit with a spatial coverage factor $C_{\mathrm{f}, \max }=0.37$. At $v_{\text {helio }}=0 \mathrm{~km} \mathrm{~s}^{-1}$, the redshift is $z=0.452060$.

is fully consistent with the value obtained from the fit of the Fe I $\lambda 2719$ absorption profile. The Fe I $\lambda 3441$ line arises on the blue wing of the C IV emission line. For this feature, the ELR to continuum flux ratio is low, $x=0.22$, which implies a $C_{\mathrm{f}}$ value of 0.82 (assuming $C_{\mathrm{elr}} \simeq 0$ and $C_{\mathrm{c}}=1$ as for the Ly $\alpha$ ELR). This is close enough to 1 to account for the absence of a significant departure from the curve of growth.

In Fig. 6 we show the equivalent width ratio $r^{\prime}$ versus $C_{\mathrm{f}}$ in the partial covering models for the two Fe I $\lambda 2719$ and $\mathrm{Fe} I \lambda 3021$ transitions. The latter have very similar $\lambda f$ values, implying that i) the allowed $\left(r_{\min }^{\prime}, r_{\max }^{\prime}\right)$ range for $C_{\mathrm{f}}=1$ is very small, and that ii) $r^{\prime}$ depends very little on line opacities (all the curves are nearly coincident, regardless of the value of $\left.\tau_{0}(\mathrm{Fe} \mathrm{I} \lambda 3021)\right)$. The observed ratio, $r^{\prime}=0.32 \pm 0.05$, lies well below the possible range for $C_{\mathrm{f}}=1$, showing unambiguously that $C_{\mathrm{f}}<1$ for this system; the inferred $C_{\mathrm{f}}$ value (corresponding to the blue cross in Fig. 6, where the line $r^{\prime}=0.32$ intersects theoretical curves) is close to the minimum covering factor associated with $C_{\mathrm{elr}}=0$, in agreement with the optimal value derived from line fitting. This figure illustrates that detecting two transitions with similar opacities, one over a quasar emission line and the other against the continuum alone, is a powerful way to establish the reality of partial covering effects.

The Fe I $\lambda 3441$ transition, which is on the C IV emission line, is intrinsically too weak to detect a significant partial covering effect. As mentioned above, its $\mathrm{W}$ measurement lies barely

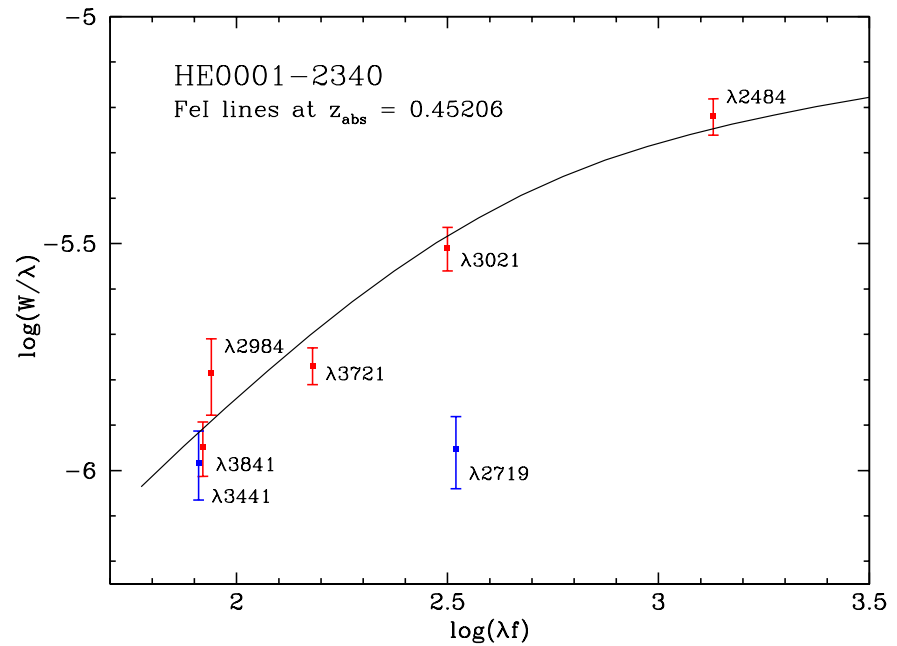

Fig. 5. Curve of growth for the Fe I transitions detected in the $z_{\mathrm{abs}}=$ 0.45206 system towards HE 0001-2340. For each point we indicate the rest wavelength of the Fe I transition involved. The smooth curve corresponds to $b=0.55 \mathrm{~km} \mathrm{~s}^{-1}$ and $\log N(\mathrm{Fe} \mathrm{I})=12.289$ (see text). The red and blue points correspond to transitions seen on the quasar continuum and emission lines, respectively. The Fe I $\lambda 2719$ line falls near the top of the quasar Ly $\alpha$ emission and is characterised by a high value of the ELR to continuum flux ratio $(x=2.31)$. We note that the corresponding $W$ measurement lies well below the curve-of-growth expectation because of partial covering of the ELR. The Fe $\mathrm{I} \lambda 3441$ is characterised by a low $x$ value, and a barely significant shift is seen.

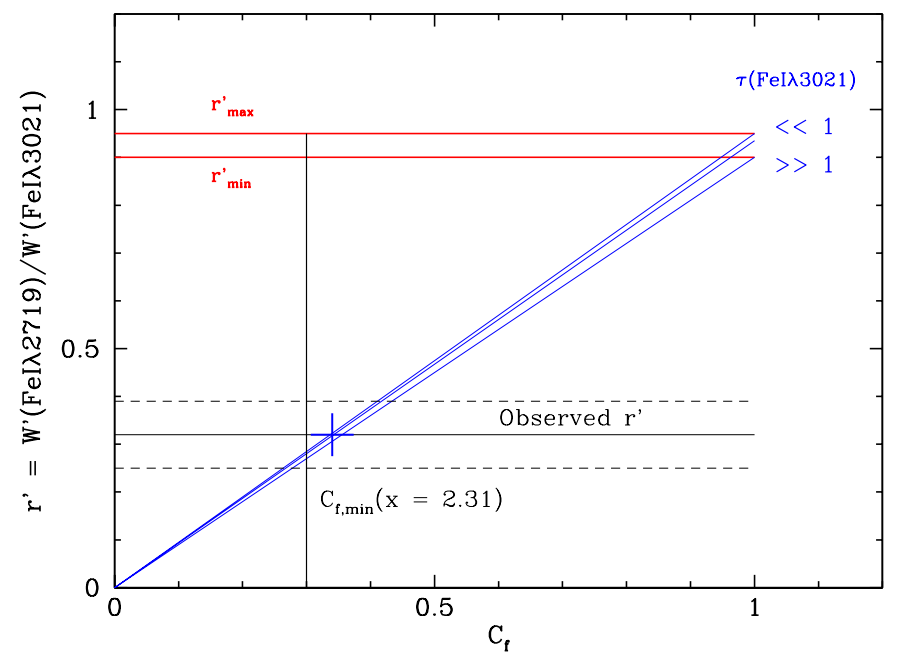

Fig. 6. Same as in Fig. 2, but for the two Fe I $\lambda 2719$ and Fe $\mathrm{I} \lambda 3021$ lines seen at $z_{\text {abs }}=0.4521$ towards HE 0001-2340. For $C_{\mathrm{f}}=1$, the range allowed for $r^{\prime}$ is very small (red horizontal lines). The behaviour of $r^{\prime}$ is shown for $\tau_{0}(\mathrm{Fe} \mathrm{I} \lambda 3021)=1, \ll 1$ and $\gg 1$ (thin blue lines); $r^{\prime}$ depends very little on line opacities. The observed ratio, $r^{\prime}=0.32 \pm 0.07$ (plain black horizontal line, with $1 \sigma$ uncertainties shown as dotted lines) is clearly outside the allowed range. It implies a value $C_{\mathrm{f}} \approx 0.33$ (thick blue cross), compatible with $C_{\mathrm{f}}=C_{\mathrm{f}, \min }$ thus $C_{\mathrm{elr}}=0$ (thick black vertical line), irrespective of $\tau_{0}(\mathrm{Fe} \mathrm{I} \lambda 3021)$.

below the curve of growth expectation for full spatial covering. In addition, nearly equally good fits were obtained when we added this transition to the three that fall on the quasar continuum assuming either $C_{\mathrm{f}}=1$ or 0.88 (i.e., $C_{\mathrm{elr}}=0.10$ ); these fits have very similar values of $N(\mathrm{Fe} \mathrm{I})$ and $b$ (the difference is about $5 \%$ ).

The Mg II doublet associated with the Fe I system at $z_{\text {abs }}=$ 0.45206 falls on the red wing of the $\mathrm{NV}$ emission line. 
Jones et al. (2010) found evidence of partial covering in this $\mathrm{Mg}$ II system and estimated $C_{\mathrm{f}} \approx 0.6$. As discussed in Sect. 2 , spatial covering effects are difficult to ascertain in such an unsaturated system because both transitions fall in a small wavelength range and thus have nearly the same associated $x$ values (we estimate $x=0.92$ at $\mathrm{Mg}$ II $\lambda 2796$ ). Furthermore, since the LFR and $W$ ratio lie in the allowed range for $C_{\mathrm{f}}=1$, it should be possible to obtain an acceptable fit that is consistent with $C_{\mathrm{f}}=1$. Using the procedure described above, we obtain a good fit of this doublet assuming full coverage: $N(\mathrm{Mg}$ II $)=$ $(1.41 \pm 0.03) \times 10^{12} \mathrm{~cm}^{-2}$ and $b=2.50 \pm 0.10 \mathrm{~km} \mathrm{~s}^{-1}$ (which corresponds to $\tau_{0}(\mathrm{Mg}$ II $\left.\lambda 2796)=1.5\right)$. Equally good fits are achieved down to $C_{\mathrm{f}}=0.75$, which leads to a possible range of the ELR coverage factor, $0.48 \lesssim C_{\text {elr }} \leq 1.0$.

\subsubsection{PKS 0237-23: the $C I$ system at $z_{\text {abs }}=1.3650$}

Srianand et al. (2007) analysed UV (IUE) and $21 \mathrm{~cm}$ (GMRT) data to derive $N(\mathrm{HI})$ for this absorber and concluded that this system is a sub-DLA. They also derived abundances by simultaneously analysing (same $z_{\mathrm{abs}}$ and $b$ ) neutral and singly-ionised species; very many components were included in their fit, and the $b$ values they derived for the $\mathrm{C}$ I components are in the range $2.5-6.8 \mathrm{~km} \mathrm{~s}^{-1}$.

This is a case for which there are only two available C I transitions, each with multiple absorption components: C I $\lambda 1656$, which falls at the top of $\operatorname{Ly} \alpha$ emission, and $\mathrm{C} I \lambda 1560$, which is in a fairly clean region with high $\mathrm{S} / \mathrm{N}$ of the Ly $\alpha$ forest. Our fit of the regions around these two transitions includes a C IV $\lambda 1550$ absorption at $z_{\mathrm{abs}}=1.52580$ bluewards of $\mathrm{C} \mathrm{I} \lambda 1656$, and a weak somewhat broad Ly $\alpha$ absorption at $z_{\mathrm{abs}}=2.22263$ (this Ly $\alpha$ absorption accounts for some of the C I components included in the Srianand et al. paper mentioned above) as well as another weak somewhat broad $\operatorname{Ly} \alpha$ absorption at $z_{\mathrm{abs}}=2.03427$, bluewards of CI $\lambda 1560$. For the UVES 2001-2002 data, the fit for the main isolated component at $z=1.364695$ and full spatial covering yields $N(\mathrm{CI})=(9.0 \pm 0.2) \times 10^{12} \mathrm{~cm}^{-2}$ and $b=4.27 \pm 0.17 \mathrm{~km} \mathrm{~s}^{-1}$, which means that the line is partly resolved. This fit is shown in Fig. 7 (red curve). It is not entirely satisfactory at least for the main isolated component: the transition on the $\operatorname{Ly} \alpha$ emission line is overfitted and the transition on the quasar continuum is underfitted, which suggests partial covering. For the other $\mathrm{C} I, \mathrm{CI}^{\star}{ }^{\star}$ components (weak and mostly blended), the discrepancy between the observation and the fit is not as conspicuous.

We then examined the possibility of a partial covering effect and applied a $C_{\mathrm{f}}$ correction factor to the normalised flux of the Ly $\alpha$ emission region. The best fit, obtained for the minimum $\chi^{2}$ value, yields $C_{\mathrm{f}}=0.85( \pm 0.05)$. For the isolated component at $z_{\mathrm{abs}}=1.364695$, we obtain $N(\mathrm{CI})=(1.05 \pm 0.02) \times 10^{13} \mathrm{~cm}^{-2}$ and $b=4.09 \pm 0.15 \mathrm{~km} \mathrm{~s}^{-1}$. The difference in the $N$ values between $C_{\mathrm{f}}=1$ and 0.85 is significant at the $5 \sigma$ level. This fit with partial covering is also good for all the other weaker $\mathrm{CI}, \mathrm{CI}^{\star}$ components. The $C_{\mathrm{f}}$ value is consistent with the value obtained for the more recent UVES data (2011-2013), although this spectrum has a lower $\mathrm{S} / \mathrm{N}$ ratio. The ratio of ELR to quasar continuum flux at $\mathrm{C} I \lambda 1656$ equals $x=2.16$, which implies a coverage factor of the ELR of about $C_{\mathrm{elr}} \sim 0.78$.

An alternative model, which assumes full spatial covering of the ELR, involves a narrow additional component that is fully blended with the strong isolated component at $z_{\mathrm{abs}}=1.364695$. The fit of this blend is poorly constrained, especially since the additional component has to be very narrow. A possible fit is $N(\mathrm{CI})=(1.71$ and 0.54$) \times 10^{13} \mathrm{~cm}^{-2}$ and $b=(0.5$

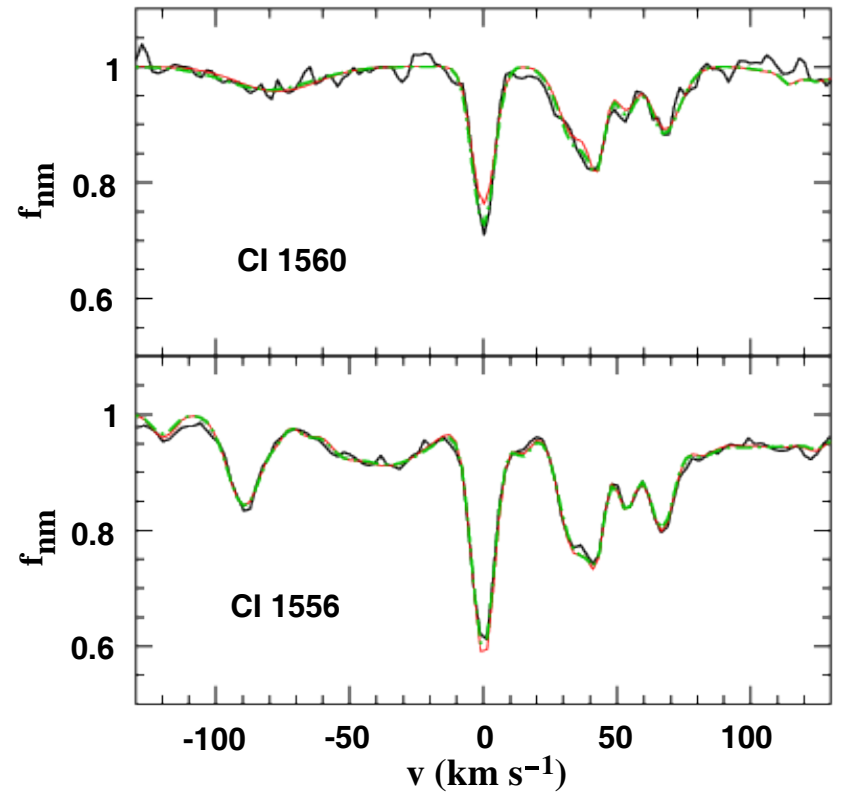

Fig. 7. Intervening $C I$ absorption towards the quasar PKS 0237-23: spectrum (black curve) and simultaneous fit to the two transitions (red curve). The green curves correspond to a fit with a spatial coverage factor $C_{\mathrm{f}}=0.85$ of the Ly $\alpha$ emission line. In the bottom panel, bluewards of C I $\lambda 1560$ line (which falls at the top of Ly $\alpha$ emission) C IV $\lambda 1550$ absorption at $z_{\mathrm{abs}}=1.52580$ is present as well as a weak, somewhat broad Ly $\alpha$ absorption. At $v_{\text {helio }}=0 \mathrm{~km} \mathrm{~s}^{-1}$, the redshift is $z=1.364695$.

and 6.4) $\mathrm{km} \mathrm{s}^{-1}$. Although such a narrow component is not unrealistic, as indeed outlined below for the $\mathrm{C}$ I absorber towards TXS $1331+170$, this alternative model is not favoured considering the fairly high temperature $(T \gtrsim 1000 \mathrm{~K})$ inferred from the detailed analysis of Srianand et al. (2007: the discussion of the velocity range $\mathrm{B}$ ).

The associated Fe II absorption is highly multiple (13 components). The Fe II $\lambda 1608$ line is in the Ly $\alpha$ forest, the Fe II triplet is on the continuum redwards of the CIV emission, and the Fe II $\lambda 2586,2600$ doublet falls on the blue wing of the C III] emission line (the Fe II $\lambda 2600$ absorption is at the top of the emission line). The component associated with the $\mathrm{C}$ I absorber is of moderate strength. For the component at $z_{\mathrm{abs}}=1.364994$, the Fe II $\lambda 2382,2600$ lines are just about saturated. The fit obtained for the five transitions redwards of the Ly $\alpha$ emission with full spatial covering is very good for all the components.

\subsubsection{Tol 0453-423: the Fe II system at $z_{\mathrm{abs}}=0.7261$}

This bright quasar has been extensively used to study its numerous absorption systems at $z_{\text {abs }}>1$ (e.g., Sargent et al. 1979; Kim et al. 2013), but a detailed study of the $z_{\mathrm{abs}}=0.7261$ system has not yet been performed.

For our analysis we used the 2002 UVES spectrum, which has high $\mathrm{S} / \mathrm{N}$. The Fe II $\lambda 2586,2600$ doublet of the $z_{\mathrm{abs}}=$ 0.7261 system falls on the quasar $\operatorname{Ly} \alpha-\mathrm{N} v$ emission. The profile of the Fe II $\lambda 2600$ absorption is extremely unusual for a singlyionised species: as clearly seen in Fig. 8 (top panel), it has a flat bottom, covering about $60 \mathrm{~km} \mathrm{~s}^{-1}$, which does not reach the zero flux level. The flux residual is at the $2.0 \%$ level; with an rms of 0.003 , this yields a detection at a $7 \sigma$ significance level. The fit obtained with nine components and full spatial covering is inconsistent with the data since the bottom of the Fe II 22600 absorption should then reach the zero flux level (red 


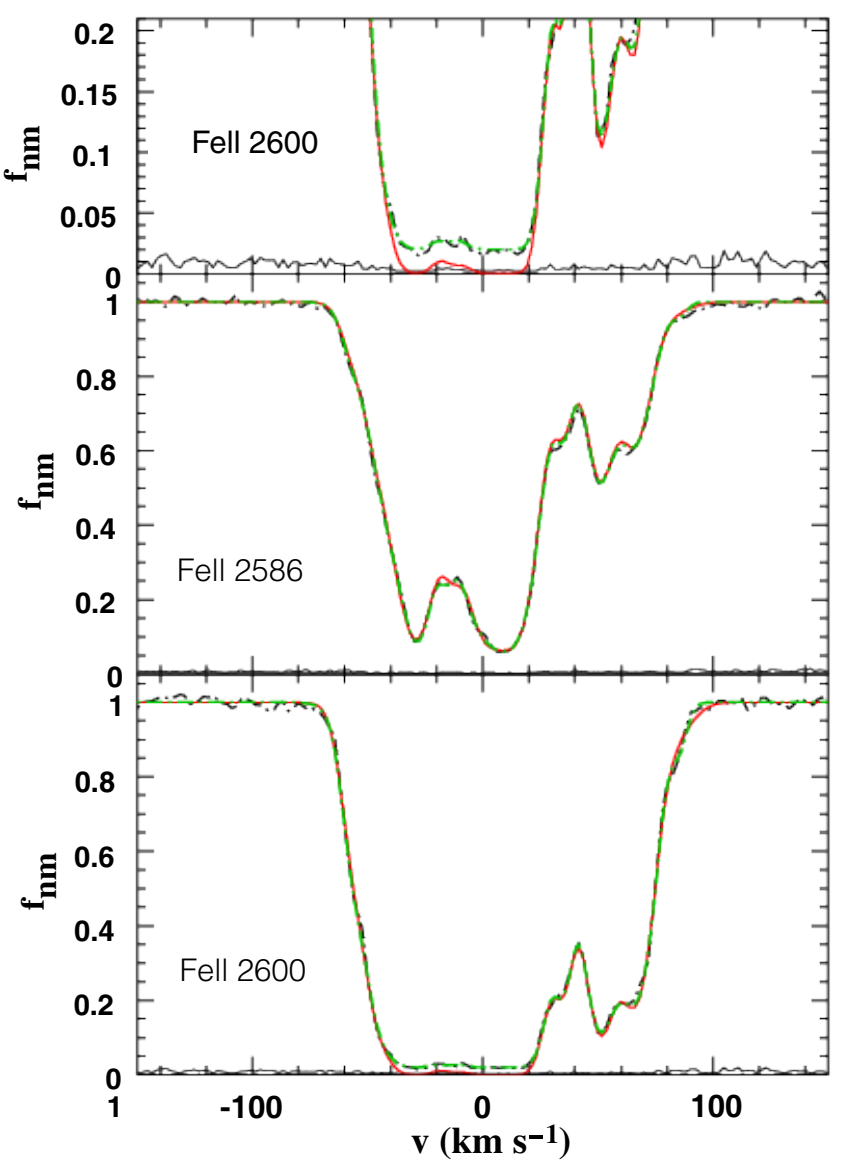

Fig. 8. Intervening Fe II absorption towards the quasar Tol 0453-423: spectrum (black curve) and simultaneous fit to the two transitions (red curve), both falling on the quasar Ly $\alpha$ emission line. The bottom black curve in each panel corresponds to the noise level. The upper panel is a zoom of the lower to clearly show the good fit of the observed spectrum as obtained with a spatial coverage factor $C_{\mathrm{f}}=0.98$ (green curve). At $v_{\text {helio }}=0 \mathrm{~km} \mathrm{~s}^{-1}$, the redshift is $z=0.72604$.

curve in Fig. 8). Although the 2011 UVES spectrum has a lower $\mathrm{S} / \mathrm{N}$, a similar residual is observed for the Fe II $\lambda 2600$ absorption (clearer after some smoothing of the data). This effect is very rarely detected for singly-ionised species; another clear example involving Si II towards LBQS $1232+082$ is discussed by Balashev et al. (2011).

The derived value of the spatial coverage factor for this Fe II absorber is $C_{\mathrm{f}}=0.98$, which for a flux ratio $x=1.22$ gives a coverage factor of the ELR of $C_{\text {elr }}=0.96$. The absorber fully covers the quasar continuum since the Fe II $\lambda 2382$ absorption line, which is in a clean part of the Ly $\alpha$ forest, reaches the zero flux level over $100 \mathrm{~km} \mathrm{~s}^{-1}$.

The Mn II absorption lines associated with this Fe II system are weak with blended components. The Mn II $\lambda 2576$ absorption line is at the top of the Ly $\alpha$ emission line and Mn II $\lambda 2606$ is at the knee of the Ly $\alpha-\mathrm{N} \mathrm{V}$ emission. Only the five components that trace the saturated part of the Fe II $\lambda 2600$ absorption line have Mn II counterparts. A good fit of the three Mn II transitions is obtained with $C_{\mathrm{f}}=1.0$. Assuming that Mn II transitions trace the same region as those of Fe II, that is, adopting the same value of $C_{\text {elr }}$, we can estimate the values of $C_{\mathrm{f}}$ for Mn II $\lambda 2576$ and 2606. Despite the large difference in the values of the flux ratios, $x(\lambda 2576,2606)=3.92,1.03$, the derived values of $C_{\mathrm{f}}$ are nearly identical $C_{\mathrm{f}}(\lambda 2576,2606)=0.97,0.98$. This was expected since $C_{\mathrm{f}}(\mathrm{Fe}$ II $)$ is very close to unity.

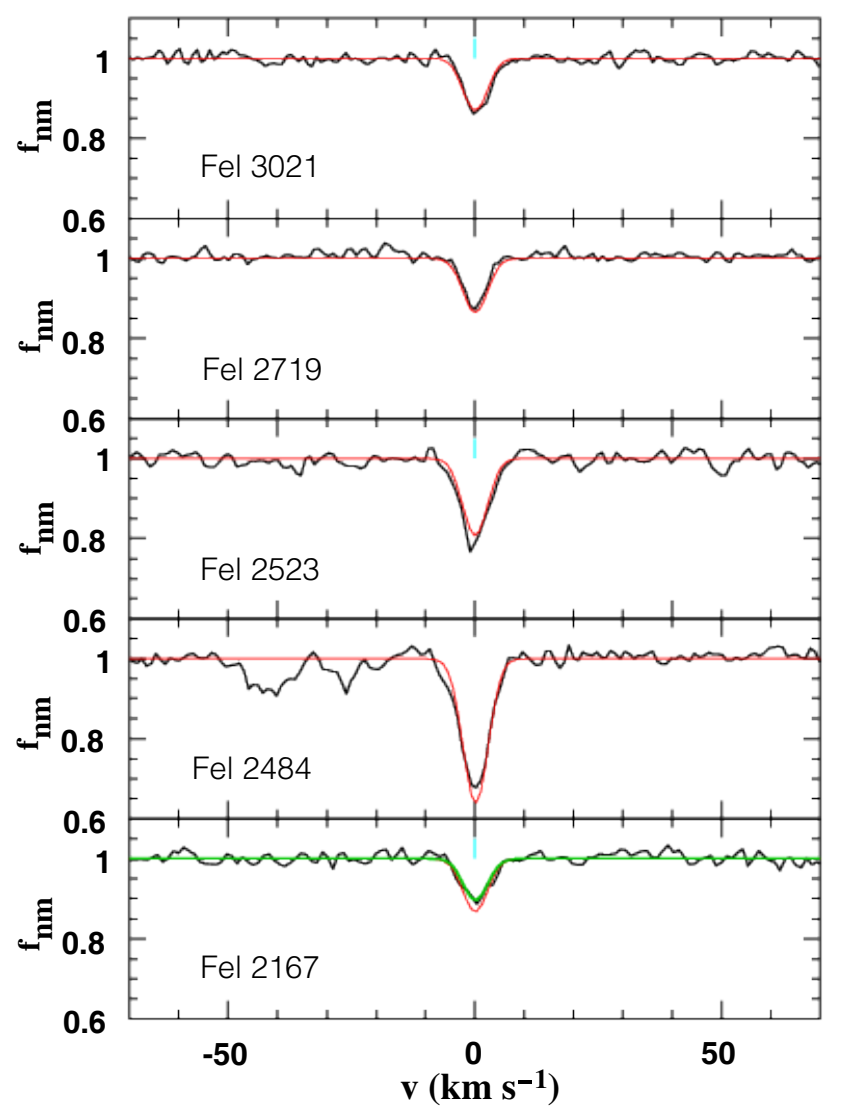

Fig. 9. Intervening Fe I absorption towards the quasar TXS 1331+170: spectrum (black curve) and simultaneous fit to the four transitions (red curve), excluding from the fit FeI2167, which falls on the quasar Ly $\alpha$ emission line. The green curve for the $\mathrm{Fe} \mathrm{I} \lambda 2167$ absorption corresponds to a fit with a spatial coverage factor $C_{\mathrm{f}}=0.8$. At $v_{\text {helio }}=0 \mathrm{~km} \mathrm{~s}^{-1}$, the redshift is $z=0.74461$.

\subsubsection{TXS $1331+170$ : the Fe I system at $z_{\text {abs }}=0.74461$}

Carswell et al. (2011) studied this quasar, but did not discuss this system. The 2011 UVES spectrum has high S/N and high spectral resolution $(F W H M=5.5 \AA)$. There are four well-detected Fe I transitions on the quasar continuum as well as the Fe I $\lambda 2167$ absorption $(\mathrm{S} / \mathrm{N} \simeq 50)$, which falls on the knee of the quasar Ly $\alpha$-N V emission. The HIRES spectrum does not cover the Ly $\alpha$ emission region, but the other four transitions on the quasar continuum (Fe I $22484,2523,2719,3021)$ are well detected. The fit of these transitions involves only one component, and within the uncertainties, the results are identical for the two spectra; for UVES we obtain $N(\mathrm{Fe} \mathrm{I})=(1.13 \pm 0.03) \times 10^{12} \mathrm{~cm}^{-2}$ and $b=1.18 \pm 0.08 \mathrm{~km} \mathrm{~s}^{-1}$, and for HIRES $N(\mathrm{Fe} \mathrm{I})=(1.12 \pm 0.05) \times$ $10^{12} \mathrm{~cm}^{-2}$ and $b=1.06 \pm 0.12 \mathrm{~km} \mathrm{~s}^{-1}$.

The Fe I $\lambda 2167$ absorption is weak (see Fig. 9) and somewhat overfitted with the $N, b$ values derived for the four transitions on the quasar UVES continuum. Its normalised minimum flux is also equal to that of the Fe I $\lambda 2719$ absorption, whereas its oscillator strength is greater than that of Fe I $\lambda 2719$ by $23 \%$. This suggests some spatial covering effect. From the minimum $\chi^{2}$ value of the fit we obtain $C_{\mathrm{f}} \sim 0.8$; this fit is also shown in Fig. 9 (green curve). The flux ratio $x$ is determined from the UVES data and equals $x=0.83$ at the position of the Fe I $\lambda 2167$ absorption, implying a coverage factor of the ELR $C_{\text {elr }} \sim 0.56$. However, this is uncertain since for a full covering of the ELR, the difference between the data and the fit is only about 1.8 times the value of 


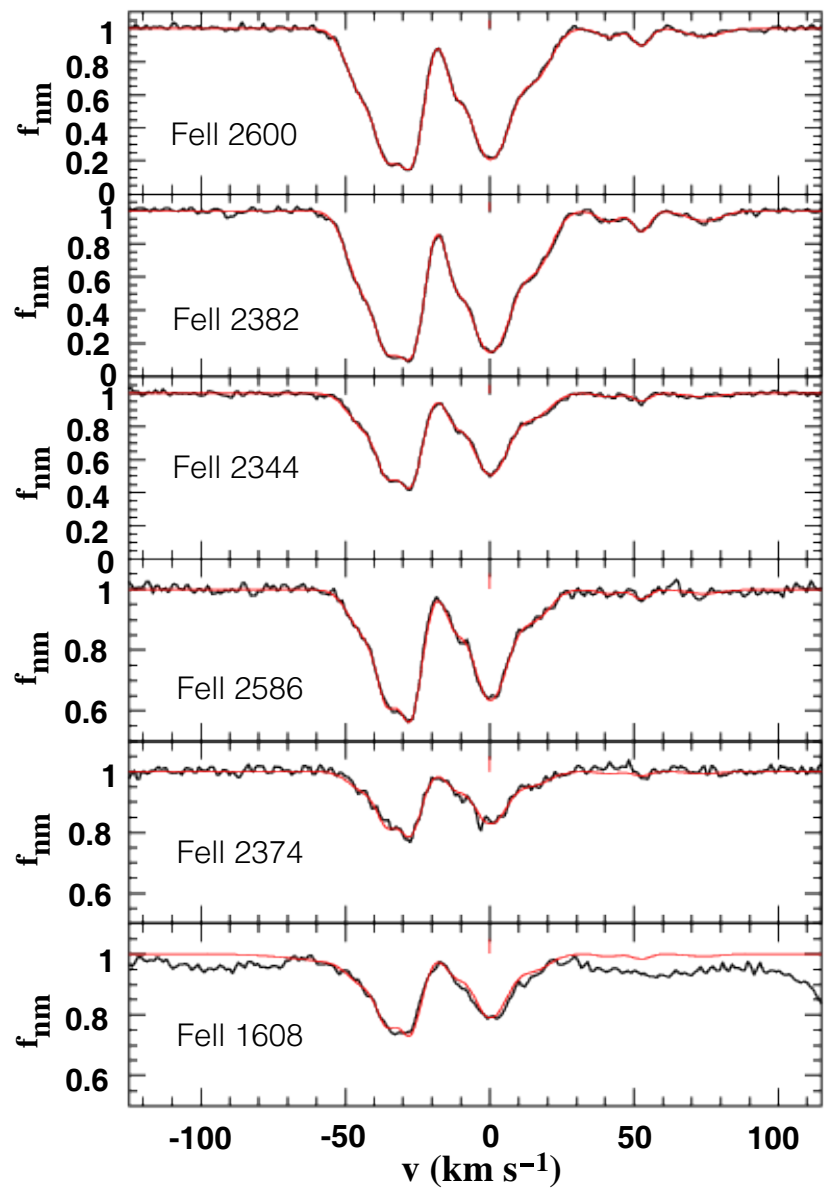

Fig. 10. Intervening Fe II absorption towards the quasar TXS 1331+170: spectrum (black curve) and simultaneous fit to the six transitions (red curve). The lower three panels correspond to Fe II lines of smaller oscillator strength and have a different $f_{\mathrm{nm}}$ scale. The Fe II $\lambda 1608$ absorptions are redshifted on top of the quasar $\operatorname{Ly} \alpha$ emission, and there are weak Ly $\alpha$ absorptions not included in the fit. At $v_{\text {helio }}=0 \mathrm{~km} \mathrm{~s}^{-1}$, the redshift is $z=1.328522$.

the spectrum rms. A full covering of the ELR therefore cannot be ruled out.

\subsubsection{TXS $1331+170$ : the Fe II system at $z_{\text {abs }}=1.32828$}

The Fe II $\lambda 1608$ absorption line of the $z_{\text {abs }}=1.32828$ system is redshifted on top of the quasar Ly $\alpha$ emission (only covered by the UVES spectrum). The other five strong Fe II lines are on the quasar continuum and well detected in the UVES spectrum (there is a defect in the HIRES data at the position of Fe II $\lambda 2382$ ). At the position of the Fe II $\lambda 1608$ absorption, the flux ratio equals $x \simeq 1.0$. There are two weak Ly $\alpha$ absorption lines, one on each side of this line (see Fig. 10). With the fit obtained for full spatial covering and nine blended subcomponents, the Fe II $\lambda 1608$ absorption is underfitted. A most likely explanation is some blending with a weak Ly $\alpha$ absorption. A good fit is indeed obtained with the addition of a weak blended $\operatorname{Ly} \alpha$ absorption component.

\subsubsection{TXS $1331+170$ : the $\mathrm{Cl}$ system at $z_{\mathrm{abs}}=1.7764$}

The neutral and singly-ionised species of this system were analysed in detail by Carswell et al. (2011), including H I (optical and $21 \mathrm{~cm}$ data), $\mathrm{H}_{2}$, and $\mathrm{C}$ I. Old UVES (2002 and 2003) and HIRES data taken at different epochs were combined for this purpose.

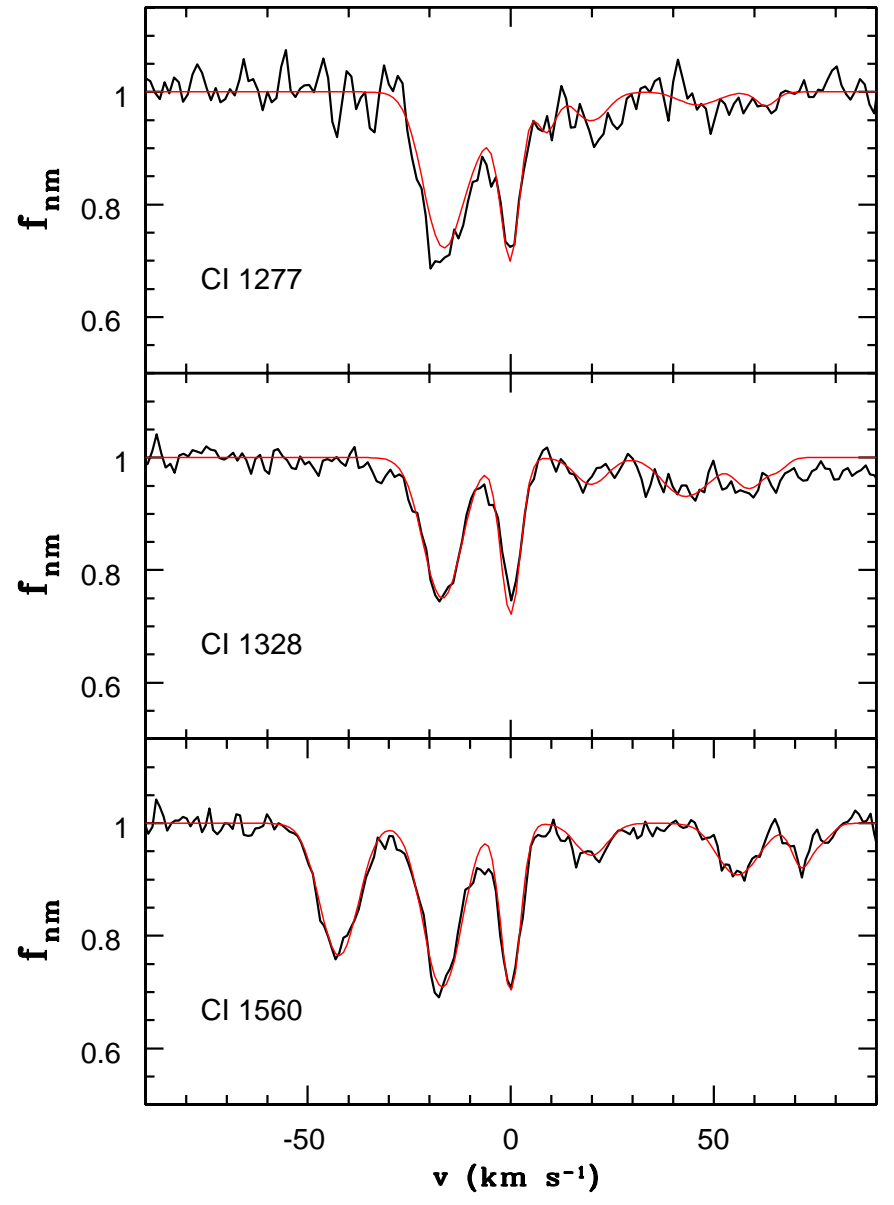

Fig. 11. Intervening C I absorption towards the quasar TXS 1331+170: spectrum (black curve) and simultaneous fit to the three transitions (red curve). In the lower panel, bluewards of C I $\lambda 1560$, Al III $\lambda 1862$ absorption is detected at $z_{\text {abs }}=1.325342$. At $v_{\text {helio }}=0 \mathrm{~km} \mathrm{~s}^{-1}$, the redshift is $z=1.776525$ : this is the most narrow $\mathrm{C}$ I component $\left(b=0.68 \mathrm{~km} \mathrm{~s}^{-1}\right)$.

One of the three detected $\mathrm{CI}$ components (at $\left.z_{\mathrm{abs}}=1.77653\right)$ is very narrow, with $b=0.55 \pm 0.13 \mathrm{~km} \mathrm{~s}^{-1}$, thus of low kinetic temperature, as confirmed by a curve-of-growth analysis. These authors discussed the possibility that this $\mathrm{C}$ I cold absorber only partially covers the background source; they concluded that it is most unlikely since the corresponding saturated $\mathrm{H}_{2}$ transitions have flat cores with zero residual intensities. We note that this is also the case for $\mathrm{O} I \lambda 1302$ in the Ly $\alpha$ forest and C II $\lambda 1334$ on the blue side of the $\operatorname{Ly} \alpha$ emission line.

Only three transitions are well detected in the 2011 UVES spectrum: $\mathrm{CI} \lambda 1277$ is in a clean region of the $\mathrm{Ly} \alpha$ forest, $\mathrm{CI} \lambda 1328$ is on the blue wing of $\operatorname{Ly} \alpha$ emission, and $\mathrm{CI} \lambda 1560$ is on the weak Si IV emission. The 1994 HIRES spectrum only covers the $\mathrm{C} I \lambda 1560,1656$ transitions. It has a somewhat lower resolution $\left(F H W M=6.25 \mathrm{~km} \mathrm{~s}^{-1}\right)$ than the 2011 UVES spectrum $\left(F H W M=5.5 \mathrm{~km} \mathrm{~s}^{-1}\right)$; we therefore did not combine these spectra for data analysis. For a full spatial covering of the background source, we find a low $b$ value for the $z_{\text {abs }}=1.77653$ component: $b=0.68 \pm 0.18$ and $0.82 \pm 0.49 \mathrm{~km} \mathrm{~s}^{-1}$ for the UVES and HIRES spectra, respectively, both with about the same column density $N(\mathrm{CI})=(1.10 \pm 0.18) \times 10^{13} \mathrm{~cm}^{-2}$. This is consistent with the results of Carswell et al. The UVES data and their fit are shown in Fig. 11; the fit includes Al III $\lambda 1862$ absorption at $z_{\mathrm{abs}}=1.325342$ bluewards of C I $\lambda 1560$.

Constraints on the spatial covering of the ELR by the cold component can only be obtained from the UVES data; we note 


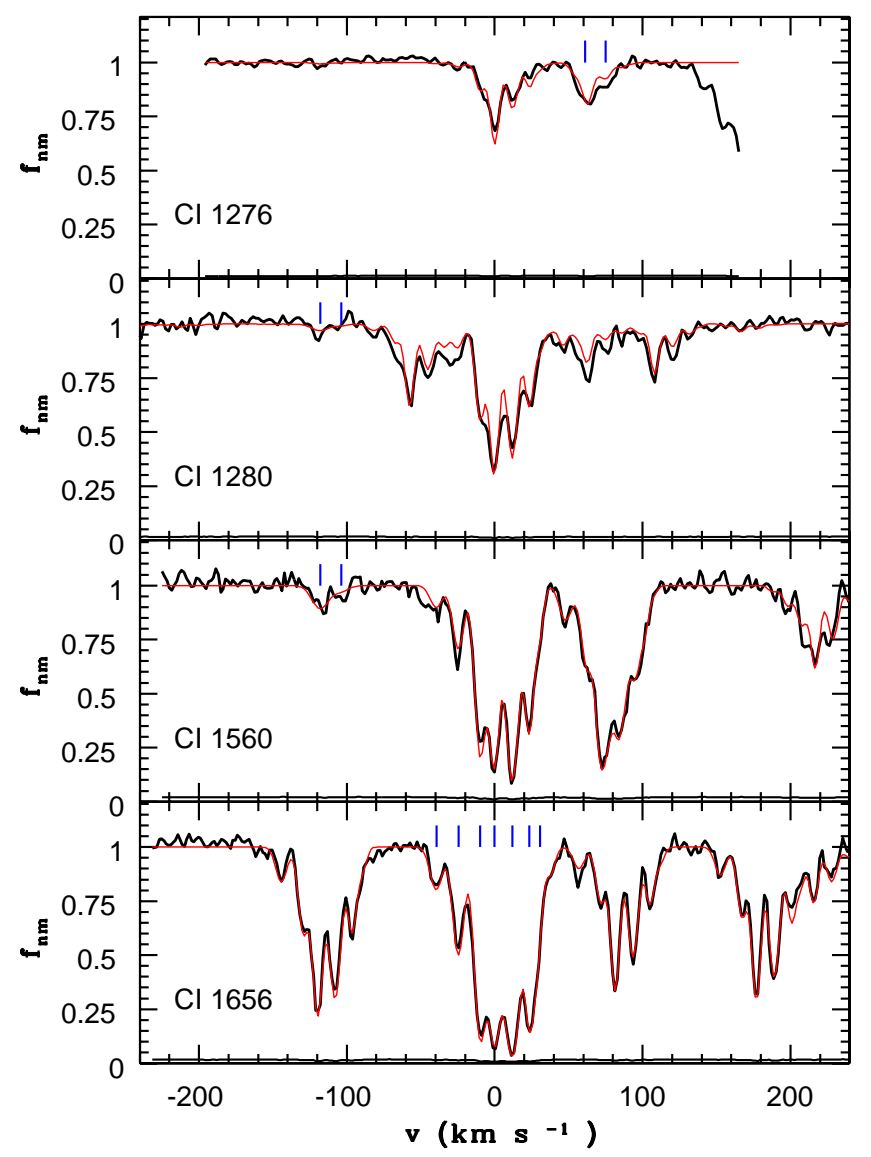

Fig. 12. Intervening $C_{I}$ absorption towards the quasar J1439+1117: spectrum (black curve) and simultaneous fit to the four transitions (red curve). The bottom black curve in each panel corresponds to the low noise level $(\mathrm{rms} \simeq 1.7 \%)$. At $v_{\text {helio }}=0 \mathrm{~km} \mathrm{~s}^{-1}$, the redshift is $z=2.418372$ : the corresponding $\mathrm{C}$ I component has the highest column density. The blue tick marks in the C I $\lambda 1656$ panel (lower one) correspond to the stronger seven components. In the $\mathrm{C}$ I $\lambda 1560$ panel, the two tick marks correspond to weaker components at -104 and $-118 \mathrm{~km} \mathrm{~s}^{-1}$ In the upper panel, the absorption seen in the $50-90 \mathrm{~km} \mathrm{~s}^{-1}$ velocity range is a blend of $\mathrm{CI}^{\star} \lambda 1276$ and $\mathrm{C} \mathrm{I} \lambda 1277$ from the two weak components with the bluest velocity.

that the spatial coverage factor should be close to unity since the difference between the observations and the fit $(\sim 1.7$ times the rms) for the C $\mathrm{\lambda} \lambda 1328$ absorption only indicates a slight overfitting. Acceptable fits can also be obtained with a coverage factor for the $\operatorname{Ly} \alpha$ emission region $C_{\mathrm{f}} \neq 1$ provided that $C_{\mathrm{f}} \gtrsim 0.9$ for the $\mathrm{C}$ I component at $z_{\mathrm{abs}}=1.77653$. The flux ratio at the position of the $\mathrm{C} I \lambda 1328$ transition equals $x=0.41$, which implies a minimum coverage factor of the ELR $C_{\text {elr }} \gtrsim 0.66$.

Four Ni II transitions associated with the CI system are detected at $z_{\text {abs }}=1.7764$. The Ni II $\lambda 1370$ absorption falls on the quasar Ly $\alpha-\mathrm{N}$ V emission line and Ni II $\lambda 1709$ on the blue wing of the C IV emission. One medium-weak component is located at $z_{\mathrm{abs}}=1.77640$ and three weak components redwards of this. A good fit is obtained for full spatial covering.

\subsubsection{QSO J1439+1117: the CI system at $z_{\mathrm{abs}}=2.41837$}

The UVES spectrum of this quasar has been discussed by Srianand et al. (2008), who detected $\mathrm{CO}, \mathrm{H}_{2}$, and $\mathrm{HD}$ molecules at $z_{\text {abs }}=2.4184$. Several $\mathrm{C} \mathrm{I}$ and $\mathrm{CI}^{\star}$ transitions (around 1276, 1277, and $1280 \AA$ ) fall on the red part of the Ly $\alpha$ emission line
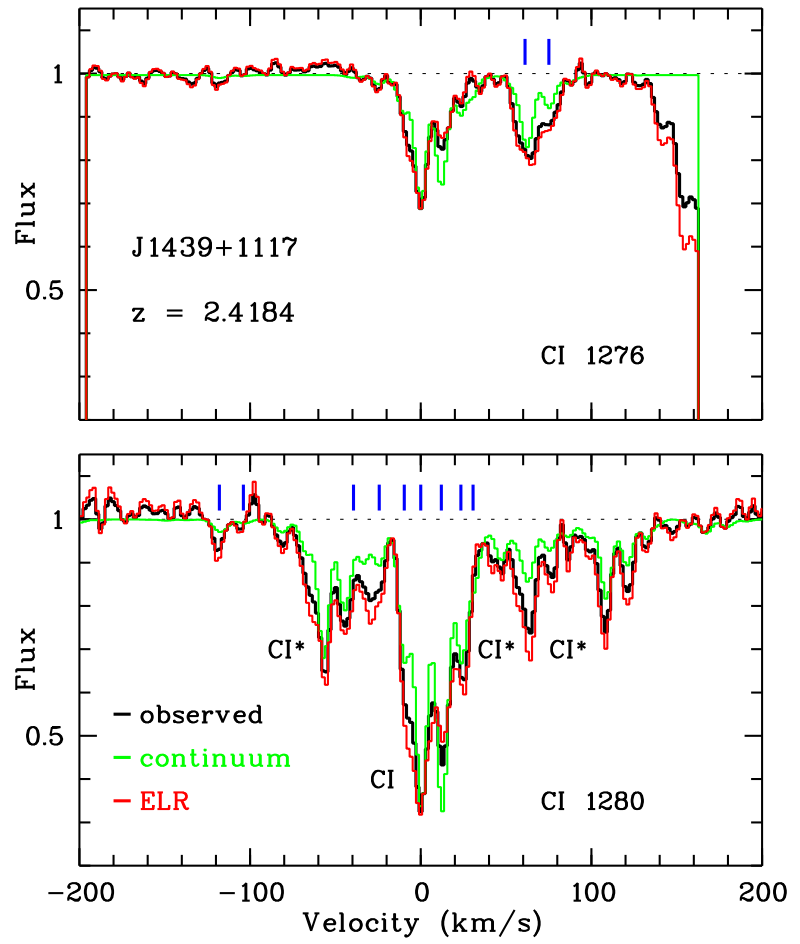

Fig. 13. Normalized profile of the $\mathrm{CI}, \mathrm{CI}^{\star}$, and $\mathrm{CI}^{\star \star}$ lines near 1276 (top panel) and $1280 \AA$ (bottom) at $z_{\text {abs }} \simeq 2.418$ towards J1439+1117, seen on the Ly $\alpha$ emission line of this quasar. Observed profiles are shown in black, while the profiles derived from fitting of the 1560 and $1656 \AA$ transitions observed against the continuum source are displayed in green and the profiles computed towards the ELR alone (see text) in red. $\mathrm{CI}^{\star}$ absorption is significantly stronger towards the ELR for the three central components (at $v=-10,0$ and $+12 \mathrm{~km} \mathrm{~s}^{-1}$ ) as well as the $\mathrm{C}$ I absorption in the $v=-10 \mathrm{~km} \mathrm{~s}^{-1}$ component. The velocity scale is the same as in Fig. 12.

while the stronger multiplets around 1560 and $1656 \AA$ are seen against the continuum source alone (unfortunately, transitions at $\lambda \approx 1328 \AA$ fall in a gap of the spectrum). We first examine whether a model in which the absorber uniformly covers the whole source is consistent with all absorption line profiles. When we attempted to fit the $\mathrm{C} I$ and $\mathrm{CI}^{\star}$ transitions near 1276, 1277, 1280,1560 , and $1656 \AA$, it clearly appeared that additional absorption is present, blended with the 1277 multiplet (it is presumably Ly $\alpha$ at $z_{\text {abs }}=2.59213$; when we adopt the emission redshift $z_{\mathrm{em}}=2.5853 \pm 0.0001$ inferred from SDSS data, this corresponds to a relative velocity of $V=-570 \mathrm{~km} \mathrm{~s}^{-1}$ ). We therefore retain only the $1276,1280,1560$, and $1656 \AA$ multiplets. The simulateneous fit of these four transitions involves seven main velocity components (indicated by blue tick marks in the bottom panel of Fig. 12) that cover a range of $70 \mathrm{~km} \mathrm{~s}^{-1}$. In addition, two weak detached components are also present at $v=-104$ and $-118 \mathrm{~km} \mathrm{~s}^{-1}$ (these can be seen in the CI 1560 panel and to a lesser extent, in the C I 1280 panel); the corresponding C I $\lambda 1277$ features are blended with $\mathrm{CI}^{\star} \lambda 1276$ absorption (upper panel of Fig. 12).

Some discrepancies between the observed spectrum and the fit are seen especially near $\mathrm{CI}^{\star}$ transitions (they are most apparent for the $1280 \AA$ multiplet), and to investigate the possibility of spatial variations over the ELR extent in more detail, we separately fit the 1560 and $1656 \AA$ multiplets (formed against the continuum source) and those at 1276,1277 , and $1280 \AA$ 
(formed against the ELR and continuum source; the Ly $\alpha$ absorption line mentioned above was included). Comparison of the two fits confirms that $\mathrm{CI}^{\star}$ absorption tends to be stronger on average towards the ELR than towards the continuum source. The total $\mathrm{CI}^{\star}{ }^{\star}$ column density derived from the 1276,1277 , and $1280 \AA$ multiplets, $N\left(\mathrm{CI}^{\star}\right)=2.16 \pm 0.11 \times 10^{14} \mathrm{~cm}^{-2}$, is significantly higher than the value derived from the 1560-1656 multiplets, $N\left(\mathrm{C} \mathrm{I}^{\star}\right)=1.50 \pm 0.04 \times 10^{14} \mathrm{~cm}^{-2}$ (the corresponding values for $\mathrm{C}$ I are nearly identical, $\left.N(\mathrm{C} \mathrm{I}) \approx 4.1 \times 10^{14} \mathrm{~cm}^{-2}\right)$. Since the two fits involve velocity components with nearly identical redshifts, it is possible to compare the absorption toward the continuum and that towards the ELR separately. To this purpose, we used Eq. (7) to extract the absorption profile toward the ELR alone for the 1276 and $1280 \AA$ transitions and adopted $x \simeq 3.1$ and 2.0, respectively, as measured on the spectrum.

The result is shown in Fig. 13, where both the ELR absorption computed from Eq. (7) (red curve) and the continuum source absorption derived from fitting the 1560 and $1656 \AA$ transitions are shown (green curve). Absorption clearly tends to be weaker towards the continuum source, especially for $\mathrm{CI}^{\star}$. By fitting the 1276 and $1280 \AA$ ELR profiles, we can compare the values of $N(\mathrm{C} \mathrm{I})$ and $N\left(\mathrm{C} \mathrm{I}^{\star}\right)$ towards the continuum source and ELR for each component. For $\mathrm{C}$ I, only the $v=-10 \mathrm{~km} \mathrm{~s}^{-1}$ component shows a significant difference, with $N(\mathrm{CI})$ larger towards the ELR by a factor $2.1 \pm 0.15$. For $\mathrm{CI}^{\star}$, the three central components at $v=-10,0$ and $+12 \mathrm{~km} \mathrm{~s}^{-1}$ display a higher column density towards the ELR by factors of $3.0 \pm 0.3,1.4 \pm 0.1$, and $2.1 \pm 0.2$, respectively. We conclude that this system shows significant spatial structure at scales in the range of $100 \mathrm{au}-0.1 \mathrm{pc}$.

\subsubsection{PKS 1448-232: the Call system at $z_{\mathrm{abs}}=-0.00002$}

The quasar PKS 1448-232 shows strong multicomponent absorption in Galactic Ca II and Na I (Ben Bekhti et al. 2008). The Ca II absorption coincides with Ly $\alpha$ and $\mathrm{N} \mathrm{V}$ emission. The two doublet lines have distinct $x$ values $(0.66$ and 0.60 for the CaII $\lambda 3934$ and 3969 lines, respectively), which offers the opportunity of investigating non-uniformity effects. Assuming that the Ly $\alpha$ ELR has an extent of about $1 \mathrm{pc}$, it delineates an angle $\theta \approx 10^{-9}$ rad or 0.20 mas, given the quasar emission redshift, $z_{\mathrm{em}}=2.208$. This corresponds to a very small linear extent of $0.02 \mathrm{au}$ in a galactic cloud located at a distance of about $100 \mathrm{pc}$. Since significant structure is detected in Galactic Ca II gas only at scales on the order of 10 au or higher (Smith et al. 2013; McEvoy et al. 2015), the absorber is expected to be uniform and provides a test case for our fitting procedure. An excellent fit is obtained for the whole Ca II doublet line profiles with $C_{\mathrm{f}}=1$, which confirms the absence of any departure from uniformity for all pieces of the Galactic gas associated with the five main velocity components.

\subsubsection{FBQS J2340-0053: the C I system at $Z_{\mathrm{abs}}=2.05454$}

There are two available spectra taken about two years apart (see Table 1). The Ci $\lambda 1560$ multiple absorption falls on the top of the CIV quasar emission line; this region is only covered by the HIRES spectrum. A good fit of the C I lines that fall on the quasar continuum (C I $\lambda 1277,1280,1328$ just redwards of the $\operatorname{Ly} \alpha-\mathrm{N}$ V quasar emission line, and $\mathrm{C}$ I $\lambda 1656$ ) is obtained separately for the HIRES and UVES spectra using eight components and assuming full coverage of the quasar continuum, $C_{\mathrm{c}}=1$. The estimated values of $N$ and $b$ are consistent between the two epochs, except for the C I component at $z_{\mathrm{abs}}=2.05472$,

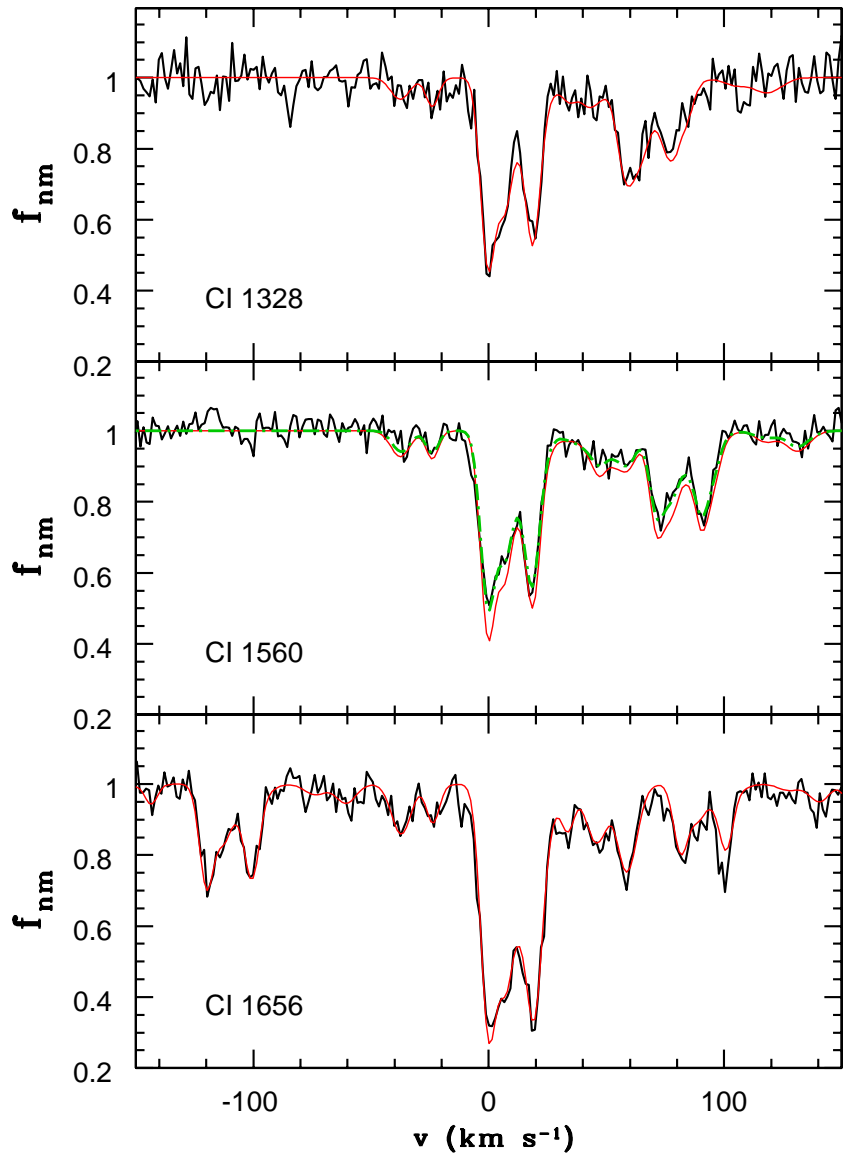

Fig. 14. Intervening $C I$ absorption towards FBQS J2340-0053: spectrum (black curve) and simultaneous fit obtained with the four transitions on the quasar continuum (red curve). The green curve for the $\mathrm{C} I$, $\mathrm{CI}^{\star} \lambda 1560$ absorptions corresponds to a fit with a spatial coverage factor $C_{\mathrm{f}}=0.85$. At $v_{\text {helio }}=0 \mathrm{~km} \mathrm{~s}^{-1}$, the redshift is $z=2.054526$.

for which tentative variation has been reported by Boissé et al. (2015).

The physical properties (density and temperature) of this multiple-component absorption system have been thoroughly investigated by Jorgenson et al. (2010) using the CI finestructure lines and $\mathrm{H}_{2}$ absorption. Their $\mathrm{C}$ I fit is solely based on the HIRES data; it includes an unidentified line blended with C I $\lambda 1328$ (Jorgenson, priv. comm.) and does not consider spatial covering effects of the C IV ELR.

Our fit of the HIRES data obtained for the four transitions that fall on the quasar continuum is shown in Fig. 14 for two transitions (C I $\lambda 1328$ and 1656) as well as for $\mathrm{C} \mathrm{I} \lambda 1560$, which is on the C IV quasar emission line. The $C \mathrm{I} \lambda 1560$ and $\mathrm{C} \mathrm{I}^{\star} \lambda 1560$ multiple-component profiles are both overfitted (Fig. 14 red curve), which points towards partial covering of the C IV emission line region. We then applied a spatial coverage factor to these absorption lines: the best fit is obtained for $C_{\mathrm{f}}=0.85$ (with an uncertainty of \pm 0.05 ), which is also plotted in Fig. 14 (green curve).

The determination of the coverage factor of the emission line region $C_{\text {elr }}$ requires an estimate of the quasar continuum flux $F_{\mathrm{c}}$ that underlies the C IV emission line. There is a 2004 SDSS lowresolution spectrum of this quasar that we use to derive a flux ratio $x=0.68$. This yields $C_{\text {elr }}=0.63$ with a possible range of $0.50<C_{\text {elr }}<0.75$. 


\subsection{Absorption systems with $\mathrm{H}_{2}$ lines on Lyß-O VI emission}

- FBQS J2340-0053, the $\mathrm{H}_{2}$ absorber at $z_{\text {abs }}=2.05473$

In this system, numerous transitions from $\mathrm{H}_{2}$ are seen from levels $J=0$ up to $J=5$ (Jorgenson et al. 2010). We examined the spectrum in detail in the range containing the quasar Ly $\beta$ $\mathrm{O}$ VI emission line and found several features from $J=0$ (at $1049.37 \AA$ ),$J=1$ (at $1049.96 \AA$ ),$J=2$ (1040.37 $\AA$ ), and $J=3$ (1041.16 and $1043.51 \AA)$ that reach the zero level at the core of absorption lines associated with the main velocity components (we estimate that the residual flux is lower than about 3\% at the $3 \sigma$ level). This unambiguously indicates that the Ly $\beta$-O VI ELR is fully covered $\left(C_{\text {elr }} \simeq 1\right)$ by both the cold molecular material $(J=0$ and $J=1)$ and the higher excitation gas $(J \geq 3)$ in these velocity components.

Other intervening systems have been studied in the literature for which some constraints can be derived. To our knowledge, the only marked partial covering effect of an ELR by an $\mathrm{H}_{2}$ absorber has been found at $z_{\mathrm{abs}}=2.338$ towards LBQS $1232+082$ (Ivanchik et al. 2010; Balashev et al. 2011). The covering factor of the O VI ELR is $C_{\text {elr }}=0.8$ (see Table 2 and Fig. 4 in Balashev et al. 2011).

For the system at $z_{\mathrm{abs}}=2.40183$ towards the quasar HE 0027-1836, Noterdaeme et al. (2007) did not discuss potential partial covering effects but presented a set of fits for $\mathrm{H}_{2}$ line profiles from levels $J=0$ up to $J=5$, including lines seen either on or off of the $\mathrm{Ly} \beta$-O VI quasar emission line (given the quasar redshift, $z_{\mathrm{em}}=2.55, \mathrm{H}_{2}$ transitions coinciding with this emission have $1075 \leq \lambda \leq 1091 \AA$ ). This is a single-component system with low $\mathrm{H}_{2}$ column density $\left(N\left(\mathrm{H}_{2}\right) \simeq 2 . \times 10^{17} \mathrm{~cm}^{-2}\right)$, and the strongest $J=0$ or $J=1$ lines are not flat-bottom and barely reach the zero level. However, no mismatch is observed in the fits for lines occurring on $\mathrm{Ly} \beta-\mathrm{O}$ VI emission, therefore we can rule out marked partial covering or structure effects.

In the $z_{\mathrm{abs}}=2.059$ system towards QSO J2123-0050, Klimenko et al. (2016) found a $3 \%$ residual flux at the core of high-opacity $J=0$ and $J=1 \mathrm{H}_{2}$ lines seen on Ly $\beta$-O VI emission, implying $C_{\text {elr }}$ is close to but significantly lower than 1 .

Finally, for the $z_{\mathrm{abs}}=2.811$ system towards PKS 0528-250 Klimenko et al. (2015) established the reality of a $2 \%$ residual flux; however, this blazar is nearly devoid of emission lines and the residual is seen regardless of the location of absorption lines in the spectrum. The interpretation favoured by these authors is that the continuum source itself is extended and includes a jet that is not entirely covered by the absorber.

\section{Estimate of the transverse absorber extent}

In the following, we discuss the implications of the results presented above about the extent and structure of the various types of the foreground absorbers we considered, involving either neutral, diffuse molecular, or moderately ionised gas. Since partial covering or structure effects involve the relative projected size of the absorber and ELR, we first need to review which information we have about the extent of the latter.

\subsection{Estimate of the ELR size}

The size-luminosity scaling relation provided by C IV reverberation mapping studies of high-redshift luminous quasars (Trevese et al. 2014, and references therein) can be used to estimate the ELR size, $l_{\mathrm{elr}}$. For instance, applying this relation to HE 0001-2340 yields an ELR overall size of about $0.3 \mathrm{pc}$. This value should be appropriate for other quasars as well since all targets in our sample have about the same luminosity and redshift, and since its dependence on luminosity $L$ is moderate (roughly $\left.\propto L^{0.5}\right)$. We note that some fraction of $\operatorname{Ly} \alpha$ emission can extend beyond the CIV ELR (Balashev et al. 2011; Fathivavsari et al. 2016, 2017).

In the following, when we discuss the constraints derived on the absorber extent, we assume for simplicity that the ELR displays a uniform brightness with the same extent at all relative velocities.

\subsection{Extent and structure of neutral absorbers}

\subsubsection{Unambiguous detections of non-uniform absorbers}

We outline the three cases with marked structure effects in neutral gas traced either by Fe I or C I.

- HE 0001-2340, the Fe I absorber at $z_{\text {abs }}=0.45206$.

This is our only clear case of small $(<50 \%)$ spatial covering of the ELR, corresponding to the configuration shown in Fig. 1a. The analysis of line profiles and the curve-of-growth method both yield a spatial coverage factor $C_{\mathrm{f}}=0.32$ of the Ly $\alpha$ ELR (see Sect. 3.2.2). This value is fully compatible with $C_{\mathrm{f}}$ being at its minimum, $C_{\mathrm{f}, \min }=0.30$ (as derived from the measured flux ratio $x$; see Figs. 5 and 6 ), that is, with a spatial coverage of the ELR $C_{\text {elr }} \simeq 0$, together with full coverage of the continuum source. An estimate of the maximum value allowed by the data is $C_{\mathrm{f}} \simeq 0.37$, implying $C_{\text {elrmax }} \simeq 0.10$. The overall extent, $l_{\mathrm{abs}}$, of the absorber must then be quite small. When we take the different redshifts of the absorber and quasar into account $\left(0.3 \mathrm{pc}\right.$ at $z_{\mathrm{em}}=2.28$ defines the same angle as $0.21 \mathrm{pc}$ at $\left.z_{\mathrm{abs}}=0.45\right), C_{\text {elr, } \max }$ scales as $\left(l_{\mathrm{abs}} / l_{\mathrm{elr}}\right)^{2}$, we obtain a maximum absorber overall size $l_{\mathrm{abs}} \simeq 0.06 \mathrm{pc}$, which is well consistent with the range 0.01-0.6pc inferred from modelling by Jones et al. (2010).

The spectroscopic characteristics of this absorber are similar to those of the so-called CaFe clouds (Bondar et al. 2007). The estimate of their physical properties depends on the assumed depletion level: either insignificant, which results in a high gas temperature $T \sim 5000-10000 \mathrm{~K}$ (Gnaciński \& Krogulec 2008), or strong, with an assumed high $\mathrm{H}_{2}$ fraction and low temperature $T<100 \mathrm{~K}$ (Jones et al. 2010). We did not detect any absorption from $\mathrm{CH}$ and $\mathrm{CH}^{+}$, although this does not exclude the presence of dust and moderate depletion, as cautioned by Welty et al. (2008).

- QSO J1439+1117, the C I absorber at $z_{\mathrm{abs}}=2.41837$.

For this system, one $\mathrm{C} I$ and three $\mathrm{CI}^{\star}$ components are found to display higher column density values towards the ELR than to the continuum source, with ratios ranging from 1.4 to 3.0. This means that significant spatial structure must be present at scales in the $100 \mathrm{au}-0.1 \mathrm{pc}$ range, with the continuum source seen through more tenuous and less dense gas at the velocities of these three components. However, since the strongest components nearly reach the zero level in $\mathrm{C} I \lambda 1277$, the associated gaseous clouds should cover the whole ELR.

- FBQS J2340-0053, the C I absorber at $z_{\text {abs }}=2.05473$.

This C I multiple absorption system has five well-detected transitions, one of which is on the C IV quasar emission line. The constraints obtained from the analysis of line profiles imply partial covering of the ELR with $C_{\text {elr }}=0.63$ for the C I and $\mathrm{CI}^{\star}$ components (see Fig. 14). This case corresponds to the configuration shown in Fig. 1b. The minimum size of the absorber, $l_{\mathrm{abs}, \min }$, is obtained when the whole absorber covers 
the ELR, for which we obtain $l_{\mathrm{abs}, \min }=0.79 l_{\mathrm{elr}}$. All values above $l_{\mathrm{abs}, \min }$ are possible for $l_{\mathrm{abs}}$. This direct constraint on the transverse size can be compared to the indirect estimate derived by Jorgenson et al. (2010) from an analysis of the physical properties of the gas (density in particular). For each main C I component (velocity range $0-25 \mathrm{~km} \mathrm{~s}^{-1}$ ) these authors give an extent along the line of sight of about $1.5 \mathrm{pc}$, which is quite consistent with our results. For such large sizes, the gas associated with the $v \simeq 19 \mathrm{~km} \mathrm{~s}^{-1}$ component is not expected to display time variability if it were uniform. Then, if the tentative variations detected by Boissé et al. (2015) between the 2006 and 2008 spectra are real, structure in the 10-100 au range must be present (we note that the velocity scale adopted in Fig. 3 from Boissé et al. is different from that in this paper).

\subsubsection{Possible detections of non-uniformity effects}

We summarize the three cases with possible spatial covering effects for neutral gas traced either by Fe I or C I.

- PKS 0237-23, the C I absorber at $z_{\mathrm{abs}}=1.36469$.

This C I multiple component absorption system has only two detected transitions: one on $\operatorname{Ly} \alpha$ emission, and the other in the $\operatorname{Ly} \alpha$ forest. The narrowest component at $z_{\mathrm{abs}}=1.36469$ is partly resolved $\left(b=4.3 \mathrm{~km} \mathrm{~s}^{-1}\right)$. The line profile analysis with partial covering of the ELR implies $C_{\mathrm{f}}=0.85$ and $C_{\text {elr }}=0.78$, thus a size $l_{\text {abs }}$ of about 0.23 pc.

An alternative model involves a "hidden" very narrow component together with full spatial covering of the ELR. The line profile analysis for this narrow component yields a width $b=0.5 \mathrm{~km} \mathrm{~s}^{-1}$; this model is not favoured considering the fairly high temperature $(T \gtrsim 1000 \mathrm{~K})$ inferred for the C I phase.

- TXS $1331+170$, the Fe I absorber at $z_{\mathrm{abs}}=0.74461$.

This Fe I single-component absorption system has five welldetected transitions, one of which is on the Ly $\alpha-\mathrm{N}$ V quasar emission line. The latter is weak and somewhat overfitted with full spatial covering. A better fit is obtained with $C_{\mathrm{f}} \sim 0.8$, thus $C_{\text {elr }}=0.56$ for $x=0.83$. However, this is not highly significant considering the small differences in the fits relative to the rms of the spectrum.

- TXS $1331+170$, the C I absorber at $z_{\text {abs }}=1.77653$.

Three well-detected transitions are present, one of which is on the blue wing of Ly $\alpha$ emission: we obtain $C_{\mathrm{f}} \gtrsim 0.9$, which for $x=0.41$ yields $C_{\text {elr }} \gtrsim 0.66$; however, $C_{\mathrm{f}}=1.0$ is fully acceptable. Consequently, this cold C I absorber does not show an unambiguous spatial covering effect, which is consistent with the presence of associated $\mathrm{H}_{2}$ saturated absorptions with flat cores and zero residual intensities.

\subsubsection{Statistical analysis}

We now attempt to extract quantitative information on the neutral absorber extent from results obtained for the whole sample. Clearly, the population is not homogeneous since the Fe I absorber at $z_{\mathrm{abs}}=0.452$ towards HE 0001-2340 covers a negligible fraction of the ELR, while the others cover all or most of it. We therefore exclude the former from our overall analysis. Although the remaining six systems provide poor statistics (we include the Ca II system towards HE 0001-2340 at $z_{\mathrm{abs}}=0.270$ since $\mathrm{Ca}$ II traces regions containing gas that is mainly neutral), the fact that only one of them shows unambiguous non-uniformity effects tells us that generally, when these absorbers cover the continuum source, they also cover most of the ELR. Their extent must then be notably larger than that of the ELR. In the following, we therefore consider that of the six absorbers that cover the continuum source, at least one displays partial covering with $C_{\text {elr }} \leq 0.75$, which leads to a probability $P\left(C_{\text {elr }} \leq 0.75\right) \geq 0.17$.

To analyse the implication of this rough estimate in terms of relative size, we assume for simplicity i) that all these six absorbers display a spherical shape with the same radius, $R_{\mathrm{a}}$; and ii) that the effect of ELR and absorber redshift differences can be ignored (we proceed as if the ELR and absorber had the same redshift). In projection, the ELR is seen as a disc (radius $R_{\mathrm{e}} \simeq$ $0.3 / 2=0.15 \mathrm{pc}$ ), and the covering factor, $C_{\mathrm{elr}}$, is simply given by the ratio $C_{\text {elr }}=A /\left(\pi R_{\mathrm{e}}^{2}\right)$, where $A$ is the area of the region over which the absorber and ELR discs overlap. Let $d$ be the projected distance between the centre of the ELR and the absorber discs. Following Ofengeim et al. (2015), $A$ can be expressed as

$A=R_{\mathrm{a}}^{2} \arccos \left(y_{\mathrm{a}}\right)+R_{\mathrm{e}}^{2} \arccos \left(y_{\mathrm{e}}\right)-H$,

with

$y_{\mathrm{a}}=\frac{d^{2}+R_{\mathrm{a}}^{2}-R_{\mathrm{e}}^{2}}{2 d R_{\mathrm{a}}}, y_{\mathrm{e}}=\frac{d^{2}+R_{\mathrm{e}}^{2}-R_{\mathrm{a}}^{2}}{2 d R_{\mathrm{e}}}$,

and

$H=\frac{\sqrt{\left(\left(R_{\mathrm{e}}+R_{\mathrm{a}}\right)^{2}-\mathrm{d}^{2}\right)\left(\mathrm{d}^{2}-\left(R_{\mathrm{a}}-R_{\mathrm{e}}\right)^{2}\right)}}{2}$.

Consider for instance a model in which $R_{\mathrm{a}}=R_{\mathrm{e}}$ and compute the expected probability of obtaining $P\left(C_{\mathrm{elr}} \leq 0.75\right)$. Of the absorbers that cover the continuum source (i.e., those that satisfy $d<R_{\mathrm{a}}$ ), Eq. (14) implies that only those with $d \geq 0.40 R_{\mathrm{e}}$ have $C_{\text {elr }} \leq 0.75$. Then, for $R_{\mathrm{a}}=R_{\mathrm{e}}$,

$P\left(C_{\text {elr }} \leq 0.75\right)=\frac{\pi R_{\mathrm{a}}^{2}-\pi\left(0.40 R_{\mathrm{e}}\right)^{2}}{\pi R_{\mathrm{a}}^{2}}=0.84$.

The latter value would imply too many absorbers with marked partial covering effects as compared to what we observe. Proceeding in the same way for higher $R_{\mathrm{a}} / R_{\mathrm{e}}$ values, we find that $P\left(C_{\text {elr }} \leq 0.75\right)=0.17$, for $R_{\mathrm{a}} \simeq 5 R_{\mathrm{e}}$. The latter value is to be considered as an upper limit for $R_{\mathrm{a}}$ since some of the systems noted "possible" might show real partial covering. We conclude that a neutral absorber radius of about $5 \times 0.15=0.75 \mathrm{pc}$ or smaller is consistent with the data. Interestingly, these values are in the range (0.2-4.7 pc) derived by Jenkins \& Tripp (2011) for Galactic C I clouds.

\subsubsection{Diffuse molecular gas}

C $\mathrm{I}$ is known to be nearly cospatial to $\mathrm{H}_{2}$ (Srianand et al. 2005), and it is therefore relevant to compare the extent derived for these two species. As discussed above (Sect. 3.3), constraints are available for very few $\mathrm{H}_{2}$ absorbers. For the only case in which a marked partial covering effect is found, the absorber towards LBQS $1232+082$, the estimated $C_{\text {elr }}$ value $(\simeq 0.7)$ is comparable to those obtained for $\mathrm{CI}$ absorbers, leading to a lower limit of a few 0.1 pc size.

It is noticeable that for the FBQS J2340-0053 absorber, the excited $\mathrm{H}_{2}(J=3)$ lines display no residual flux. This provides useful constraints for models invoking intermittent turbulent vortices to account for this warmer gas (Godard et al. 2014): although these localized regions are supposed to fill a tiny volumic fraction, their number density and extent must be such that their surface coverage factor is very close to unity. 


\subsection{Singly-ionised gas}

When possible, we searched for structure effects in moderately ionised gas, as traced by species such as Mg II, Fe II, and Mn II.

The only conclusive detection of partial covering of the ELR for a singly-ionised species is that of Fe II at $z_{\mathrm{abs}}=0.72609$ towards Tol $0453-423$. The effect is small, but very unusual: the Fe II $\lambda 2600$ saturated components have a flat core with a clear flux residual of $2 \%$ ( $7 \sigma$ significance level). We obtain from the fit of this Fe II doublet, which falls on the quasar Ly $\alpha-\mathrm{N}$ V emission, $C_{\mathrm{f}}=0.98$ and $C_{\text {elr }}=0.96$, that is, an absorber size comparable to or larger than the ELR.

For two cases, either full or partial covering of the ELR are equally acceptable or inconclusive due to the weakness of the absorption lines: the $\mathrm{Mg}$ II absorber at $z_{\mathrm{abs}}=0.452$ towards HE 0001-2340, with $0.75 \lesssim C_{\mathrm{f}} \leq 1.0$, and the very weak Mn II absorber at $z_{\text {abs }}=0.726$ towards Tol 0453-0423.

Finally, two cases are well consistent with full covering of the ELR: the multiple Fe II system at $z_{\mathrm{abs}}=1.365$ towards PKS 0237-23 and the multiple Fe II system at $z_{\mathrm{abs}}=1.328$ towards TXS 1331+170; in total 22 distinct velocity components are detected in these Fe II absorbers. The absence of structure effects in these Fe II absorbers appears to be in good agreement with constraints obtained previously concerning the extent - about $200 \mathrm{pc}$ after Rauch et al. (2002) - of low-ionisation cloudlets associated with individual velocity components.

\section{Discussion and prospects}

\subsection{Detection of non-uniformity effects}

The analysis of the systems in our sample illustrates well the difficulties encountered in establishing the reality of nonuniformity effects in foreground absorbers. Large departures from uniformity as seen towards HE 0001-2340 for Fe I at $z_{\mathrm{abs}}=0.45206$ are very rare, and the observed effects are generally too small to bring equivalent widths or apparent opacity ratios clearly out of the range expected for a point-like background source. When the values of these ratios remain within the optically thin and thick bounds, the apparent inconsistencies between the various line profiles can potentially be removed by introducing "hidden" additional velocity components (see the $z_{\mathrm{abs}}=1.364$ absorber towards PKS 0237-23: we note, however, that for this system, additional arguments do not favour the presence of a low $b$ component). In the interstellar medium of our own Galaxy, ultra-high resolution observations have directly shown that $b$ parameters in some neutral velocity components can be lower than $b=1 \mathrm{~km} \mathrm{~s}^{-1}$ (Andersson et al. 2002); our line fitting or curve-of-growth analysis also require similarly narrow lines, but for severe blending, our resolution of about $6 \mathrm{~km} \mathrm{~s}^{-1}$ might be insufficient to reach reliable conclusions.

It is noteworthy, however, that when two transitions with about equal $\lambda f$ values (e.g., to within $10 \%$ ) are detected, one against the continuum and the other one on an emission line, robust results can be obtained; indeed, the opacities being then nearly equal, line profiles are expected to be identical in the velocity scale (corresponding to $W \propto \lambda$ ), regardless of any assumption on the velocity distribution (Fe I $\lambda 3021$ and $\mathrm{Fe} I \lambda 2719$ seen towards HE 0001-2340 nearly fulfill this condition), which results in a very narrow range expected for the $W$ ratio (Fig. 6). For the main $\mathrm{CI}$ and $\mathrm{Fe} \mathrm{I}$ transitions, we note that the $\lambda f$ ratio of the $\mathrm{CI} \lambda 1277$ to $\mathrm{CI} \lambda 1328$ lines is 1.075 , while the $\lambda f$ ratios of the $\mathrm{Fe} \mathrm{I} \lambda 2719$ to $\mathrm{Fe} \mathrm{I} \lambda 2167$ lines and the $\mathrm{Fe} \mathrm{I} \lambda 2719$ to $\mathrm{Fe} \mathrm{I} \lambda 3021$ lines are 1.018 and 1.054 , respectively, which is very close to unity. This means that for a C I or Fe I system that displays one of these transitions on an emission line, it would be easier to investigate structure effects.

One obviously important parameter in detecting structure effects is the emission line over continuum contrast, that is the $x$ value. If the latter is high (as with absorption detected on top of Ly $\alpha$ emission), small departures from full covering can be revealed more easily. In practise, for the high $z$ quasars considered in this study, Ly $\alpha-\mathrm{N}$ V or C IV emission, and to a lesser extent $\mathrm{Ly} \beta$-O VI, are the only emission lines providing reasonably high $x$ values. Another key parameter is the absorption line opacity: when optically thick flat-bottom features are seen on emission lines, departures from full covering of only a few percent can be established reliably, as illustrated by the $z_{\mathrm{abs}}=0.726$ system towards Tol 0453-423. For low- or intermediate-opacity lines, the detection of several transitions with various $f$ values formed against either the continuum source or ELR is important to achieve well-constrained fits for absorption line profiles and to assess the uniformity of the absorber. Species such as Fe I and $\mathrm{C}$ I are well suited owing to the large number of relatively strong transitions they provide.

Finally, we pinpoint a few values of the $r=\left(1+z_{\mathrm{em}}\right) /\left(1+z_{\mathrm{abs}}\right)$ ratio that provide especially favourable distributions of Fe I and CI absorption lines with respect to quasar emission lines. We first consider the main $\mathrm{C}$ I transitions and we find that $r \simeq 1.072$ brings the $1260,1276,1277$, and $1280 \AA$ transitions on Ly $\alpha$ $\mathrm{N}$ V together with $\mathrm{C} I \lambda 1656$ on C IV emission (this corresponds to the system observed by Balashev et al. 2011 with $z_{\mathrm{em}}=2.57$ and $z_{\mathrm{abs}}=2.338$, thus $r=1.070$ ). For $r \simeq 1.035$, the 1260, 1276, 1277 , and $1280 \AA$ transitions lie on Ly $\alpha$-N v, while all other transitions are seen against the continuum source alone. For Fe I, we find that $r \simeq 2.512$ (Fe I $\lambda 3021$ on Ly $\alpha$ and Fe I $\lambda 3720$ on C IV), 2.239 ( $\mathrm{Fe} \mathrm{I} \lambda 2719$ on $\mathrm{Ly} \alpha$ and $\mathrm{Fe} \mathrm{I} \lambda 3441$ on $\mathrm{CIV}$, as towards HE 0001-2340) and $1.928(\mathrm{Fe} I \lambda 3021$ on $\mathrm{CIV}$ and Fe I $\lambda 3720$ on $\mathrm{C}$ III]) provide valuable configurations.

\subsection{Incomplete absorption systems}

The discussion in Sect. 4.2 and our conclusion that the extent of cold neutral absorbers is about five times that of the ELR raises the possibility of detecting absorption lines induced by cloudlets that cover part of the ELR, but not the continuum source (Fig. 1c). This would correspond to velocity components seen only on emission lines, with no corresponding feature detected in transitions seen against the continuum. Such small clouds are expected to be embedded in a more extended HI region and in an even larger ionised gaseous envelope. Thus, one does not expect to miss the identification of systems that would present absorption lines exclusively on emission lines, but instead to occasionally encounter some additional velocity components on the emission line.

The simple model described in Sect. 4.2.3 can be used to estimate the incidence of such components. The associated absorbers are characterised by $R_{\mathrm{a}}<d<R_{\mathrm{a}}+R_{\mathrm{e}}$; then, their number $\left(N_{\mathrm{e}}\right)$ relative to the number of those covering the continuum source $\left(N_{\mathrm{c}}\right)$ is given by

$$
\frac{N_{\mathrm{e}}}{N_{\mathrm{c}}}=\frac{\pi\left(R_{\mathrm{a}}+R_{\mathrm{e}}\right)^{2}-\pi R_{\mathrm{a}}^{2}}{\pi R_{\mathrm{a}}^{2}}=\left(1+\frac{R_{\mathrm{e}}}{R_{\mathrm{a}}}\right)^{2}-1 .
$$

For $R_{\mathrm{a}} \simeq 5 R_{\mathrm{e}}$, we obtain $N_{\mathrm{e}} / N_{\mathrm{c}}=0.44$. A significant fraction of incomplete systems is therefore expected, but the latter should on average exhibit much weaker features since the absorbed flux is low. $C_{\mathrm{c}}=0$, and furthermore, for $R_{\mathrm{a}}=5 R_{\mathrm{e}}$, we find that $50 \%$ 
of these systems have $0<C_{\text {elr }}<0.17$ (with $d$ lying in the range $5.52 R_{\mathrm{e}}<d<6 R_{\mathrm{e}}$ ); the remaining $50 \%$ have $0.17<C_{\text {elr }}<$ 0.48 and $5 R_{\mathrm{e}}<d<5.52 R_{\mathrm{e}}$. We searched for such additional components within the $\mathrm{CI}$ and Fe I systems investigated in this paper and could not find any. To our knowledge, no absorption feature of this type has been reported.

\subsection{Structure effects and $f$ values}

Some oscillator strength values for UV transitions that are commonly detected in quasar absorption systems are still not accurately determined. In particular, there has been some debate on the $f$ values for C I transitions as derived by Jenkins \& Tripp $(2001,2011)$ from interstellar absorption lines using high spectral resolution HST-STIS data, and those given by Morton (2003). When precise laboratory measurements or computations are not available, astrophysical data can be used to constrain the ratio of $f$ values for several transitions from a given species (see, e.g., Federman \& Zsargo 2001). The excellent S/N quasar spectra considered in this paper could help to determine which of the two sets of C I $f$ values is the most consistent with the observed line profiles.

In doing this, transitions that occur on emission lines must of course be dismissed because a change in $f$ and partial covering of the ELR might be confused. Equation (2) indicates that for low opacities, $F_{\mathrm{obs}, n}$ reduces to $F_{\mathrm{obs}, n} \simeq 1-\tau C_{\mathrm{f}}$ where $\tau \propto$ $f$; thus both effects are indistinguishable in the $\tau \ll 1$ limit. All our measurements are consistent with full covering of the continuum source, as indeed expected given the small size of the latter. Then, for transitions seen against the continuum, quasar spectra can provide reliable constraints on the ratio of $f$ values, exactly as stellar spectra do for interstellar lines.

We therefore selected pairs of $\mathrm{CI}_{\mathrm{I}}$ transitions seen on the quasar continuum, when possible in regions of similar $\mathrm{S} / \mathrm{N}$, and with Morton's $f$ ratios differing substantially from those of Jenkins \& Tripp. This is clear for the $f(\mathrm{C} I \lambda 1560) / f(\mathrm{C} \mathrm{I} \lambda 1656)$ ratio: using Jenkins \& Tripp, it is 1.74 times that obtained with Morton's $f$ values (there is a similar difference for $\mathrm{CI}^{\star}$ transitions). It is also the case, but to a lesser extent, for the $f(\mathrm{C} \mathrm{I} \lambda 1560) / f(\mathrm{C} \mathrm{I} \lambda 1277)$ ratio, which is 1.13 times higher when adopting the values of Jenkins \& Tripp instead of those of Morton.

Two systems with $\mathrm{CI} \lambda 1560$ and $\mathrm{CI} \lambda 1656$ transitions are detected against the continuum: at $z_{\mathrm{abs}}=1.77653$ towards TXS 1331+170 (HIRES spectrum), and at $z_{\mathrm{abs}}=2.41837$ towards QSO J1439+1117 (UVES spectrum). The simultaneous fit of these lines in both cases results in a much poorer fit when using the $f$ values of Jenkins \& Tripp instead of those of Morton. For TXS $1331+170$, the $\chi^{2}$ statistic for the fit is $33 \%$ higher, with the $\mathrm{C} I \lambda 1560$ transition of the moderate-opacity component at $-17 \mathrm{~km} \mathrm{~s}^{-1}$ (see Fig. 11) clearly overfitted, whereas it is underfitted for the $\mathrm{C} \mathrm{I} \lambda 1656$ transition. The system at $z_{\mathrm{abs}}=2.41837$ toward QSO J1439+1117 has multiple components with strong $\mathrm{CI}^{\star}$ absorptions of intermediate opacity. The $\chi^{2}$ statistics for the fit is $95 \%$ higher with Jenkins \& Tripp's $f$ values, corresponding to a clear overfit of the $\mathrm{CI}^{\star} \lambda 1560$ transitions and significant underfit for the $\mathrm{C}^{\star} \lambda 1656$ lines. In contrast, satisfactory fits are obtained for both systems using Morton's values.

The $\mathrm{C} \mathrm{I} \lambda 1560$ and $\mathrm{C} \mathrm{I} \lambda 1277$ transitions are present at $z_{\mathrm{abs}}=$ 1.77653 in the UVES spectrum of TXS $1331+170$. Since the $f$ ratios for these transitions only differ by $13 \%$ between Jenkins \& Tripp's and Morton's values, we do not expect a strong difference in the fits. The $\chi^{2}$ statistics for the fit is only $9 \%$ higher with Jenkins \& Tripp's $f$ values instead of Morton's values. In both cases, the fit of $\mathrm{C} I \lambda 1560$ absorptions is good (the $\mathrm{S} / \mathrm{N}$ ratio is better near the $\mathrm{C} I \lambda 1560$ lines, which drive the fit), whereas the C I $\lambda 1277$ absorption of the component at $-17 \mathrm{~km} \mathrm{~s}^{-1}$ (see above) is slightly underfitted when using Jenkins \& Tripp's $f$ values.

Therefore, the $f$ values adopted for C I in our study (from Morton 2003) appear to be more consistent with the data for the three systems mentioned above.

Another species with transitions for which $f$ values are still uncertain is Ni II (see Cassidy et al. 2016 for a comparison between calculations, experimental determinations, and observations). In the course of our study, we identified a few systems that we believe to be free of partial covering or structure effects; we plan to investigate them in a future paper in order to improve the determination of $f$ values for this ion.

\subsection{Prospects}

The conclusions drawn from our analysis are limited by the small number of systems we investigated, and it is clearly desirable to extend the $\mathrm{C}$ I and Fe I samples. Additional $\mathrm{C}$ I absorbers can be selected with an appropriate $\left(1+z_{\mathrm{abs}}\right) /\left(1+z_{\mathrm{em}}\right)$ ratio from the $\mathrm{C}$ I survey performed by Ledoux et al. (2015) on the basis of SDSSII DR9 spectroscopic data. This could be complemented by a similar CI search in the public SDSS-III DR12 spectroscopic database. For Fe I, systems involving this species are much rarer and weaker than CI systems, and it would be very difficult to build a sample of reasonable size. We note that some multiobject spectrographs such as MOONS at the VLT and 4MOST at VISTA, which are still under construction, will help in the near future to increase the number of high-redshift quasar identifications and will provide additional C I and Fe I systems of interest for studies of cold neutral absorber extent and structure.

Another promising approach would consist of using observations with higher spectral resolution. As illustrated by our study, at a resolution of about $6 \mathrm{~km} \mathrm{~s}^{-1}$, some velocity components in neutral gas remain unresolved, and furthermore, line blending of adjacent components is often a problem in establishing the reality of structure effects. The upcoming ESO instrument ESPRESSO, with its higher resolution $(R=120000$ and up to $220000)$ and full wavelength coverage from 3800 to $7800 \AA$ (Pepe et al. 2013), would clearly allow us to perform a much more detailed study of the few systems for which we have identified partial covering or non-uniformity effects. This is especially true for the complex system at $z_{\mathrm{abs}}=2.41837$ towards QSO J1439+1117, which involves many closely spaced velocity components.

Acknowledgements. We are grateful to the anonymous referee for the careful reading of the manuscript and numerous constructive comments. We also warmly thank Steve Federman, Pasquier Noterdaeme, and Serj Balashev for fruitful discussions.

\section{References}

Andersson, B.-G., Wannier, P. G., \& Crawford, I. A. 2002, MNRAS, 334, 327 Arav, N., Kaastra, J., Kriss, G. A., et al. 2005, ApJ, 620, 665 Balashev, S. A., Petitjean, P., Ivanchik, A. V., et al. 2011, MNRAS, 418, 357 Barlow, T. A., \& Sargent, W. L. W. 1997, AJ, 113, 136

Ben Bekhti, N., Richter, P., Westmeier, T., \& Murphy, M. T. 2008, A\&A, 487, 583

Bentz, M. C., Peterson, B. M., Netzer, H., et al. 2009, ApJ, 697, 160 Bergeron, J., Petitjean, P., Aracil, B., et al. 2004, The Messenger, 118, 40 Boissé, P., Bergeron, J., Prochaska, J. X., et al. 2015, A\&A, 581, A109 Bondar, A., Kozak, M. Gnaciński, G. A., et al. 2007, A\&A, 378, 893 Carswell, R. F., Jorgenson, R. A., Wolfe, A. M., \& Murphy, M. T. 2011, MNRAS, 411, 2319 
Cassidy, C. M., Hibbert, A., \& Ramsbottom, C. A. 2016, A\&A, 587, A107

Dai, X., Kochanek, C. S., Chartas, G., et al. 2010, ApJ, 709, 278

D'Odorico, V. 2007, A\&A, 470, 529

Fathivavsari, H., Petitjean, P., Noterdaeme, P., et al. 2016, MNRAS, 461, 1816

Fathivavsari, H., Petitjean, P., Zou, S., et al. 2017, MNRAS, 466, L5

Federman, S. R., \& Zsargó, J. 2001, ApJ, 555, 1020

Ganguly, R., Eracleous, J., Charlton, J. C., et al. 1999, AJ, 117, 2594

Gnaciński, P., \& Krogulec, M. 2008, A\&A, 477, 543

Godard, B., Falgarone, E., \& Pineau des Forêts, G. 2014, A\&A, 570, A27

Hamann, F., Barlow, T. A., Junkkarinen, V., \& Burbidge, E. M. 1997, ApJ, 478, 80

Hamann, F., Kanekar, N., Prochaska, J. X., et al. 2011, MNRAS, 410, 1957

Ivanchik, A. V., Petitjean, P., Balashev, S. A., et al. 2010, MNRAS, 404, 1583

Jenkins, E. B., \& Tripp, T. M. 2001, ApJS, 137, 297

Jenkins, E. B., \& Tripp, T. M. 2011, ApJ, 734, 65

Jones, T. M., Misawa, T., Charlton, J. C., et al. 2010, ApJ, 715, 1497

Jorgenson, R. A., Wolfe, A. M., \& Prochaska, J. X. 2010, ApJ, 722, 460

Kim, T.-S., Partl, A. M., Carswell, R. F., \& Müller, V. 2013, A\&A, 552, A77

Klimenko, V. V., Balashev, S. A., Ivanchik, A. V., et al. 2015, MNRAS, 448, 280

Klimenko, V. V., Balashev, S. A., Ivanchik, A. V., \& Varshalovich, D. A. 2016, AstL, 42, 137

Krogager, J.-K., Fynbo, J. P. U., Noterdaeme, P., et al. 2016, MNRAS, 455, 2698

Ledoux, C., Noterdaeme, P., Petitjean, P., \& Srianand, R. 2015, A\&A, 580, A8
McEvoy, C. M., Smoker, J. V., Dufto, P. L., et al. 2015, MNRAS, 451, 1396 Molaro, P., Centurión, M., Whitmore, J. B., et al. 2013, A\&A, 555, A68 Morton, D. C. 2003, ApJS, 149, 205

Noterdaeme, P., Ledoux, C., Petitjean, P., et al. 2007, A\&A, 474, 393

Ofengeim, D. D., Balashev, S. A., Ivanchik, A. V., Kaminker, A. D., \& Klimenko, V. V. 2015, Ap\&SS, 359, 26

Pepe, F., Cristiani, S., Rebolo, R., et al. 2013, The Messenger, 153, 6

Prochaska, J. X., Wolfe, A. M., Howk, J. C., et al. 2007, ApJS, 171, 29

Rauch, M., Sargent, W. L. W., \& Barlow, T. A. 2001, ApJ, 554, 823

Rauch, M., Sargent, W. L. W., Barlow, T. A., \& Simcoe, R. A. 2002, ApJ, 576, 45

Sargent, W. L. W., Young, P. J., Boksenberg, A., Carswell, R. F., \& Whelan, J. A. J. 1979, ApJ, 230, 49

Smith, K. T., Fossey, S. J., Cordiner, M. A., et al. 2013, MNRAS, 429, 939

Srianand, R., Petitjean, P., Ledoux, C., Ferland, G., \& Shaw, G. 2005, MNRAS, 362,549

Srianand, R., Gupta, N., \& Petitjean, P. 2007, MNRAS, 375, 584

Srianand, R., Noterdaeme, P., Ledoux, C., \& Petitjean, P. 2008, A\&A, 482, L39

Trevese, D., Perna, M., Vagnetti, F., Saturni, F. G., \& Dadina, M. 2014, ApJ, 795 , 164

Watson, J. K., \& Meyer, D. M. 1996, ApJ, 473, 127

Welty, D. E. 2007, ApJ, 668, 1012

Welty, D. E., Simon, T., \& Hobbs, L. M. 2008, MNRAS, 388, 323

Woo, S. C., Turnshek, D. A., Badenes, C., \& Bickerton, S. 2013, MNRAS, 434, 141 